\title{
Germs of arcs on singular algebraic varieties and motivic integration
}

\author{
Jan Denef $\quad$ François Loeser
}

Revised Nov. 1997, to appear in Invent. Math.

\section{Introduction}

Let $k$ be a field of characteristic zero. We denote by $\mathcal{M}$ the Grothendieck ring of algebraic varieties over $k$ (i.e. reduced separated schemes of finite type over $k$ ). It is the ring generated by symbols $[S]$, for $S$ an algebraic variety over $k$, with the relations $[S]=\left[S^{\prime}\right]$ if $S$ is isomorphic to $S^{\prime},[S]=\left[S \backslash S^{\prime}\right]+\left[S^{\prime}\right]$ if $S^{\prime}$ is closed in $S$ and $\left[S \times S^{\prime}\right]=[S]\left[S^{\prime}\right]$. Note that, for $S$ an algebraic variety over $k$, the mapping $S^{\prime} \mapsto\left[S^{\prime}\right]$ from the set of closed subvarieties of $S$ extends uniquely to a mapping $W \mapsto[W]$ from the set of constructible subsets of $S$ to $\mathcal{M}$, satisfying $\left[W \cup W^{\prime}\right]=[W]+\left[W^{\prime}\right]-\left[W \cap W^{\prime}\right]$. We set $\mathbf{L}:=\left[\mathbf{A}_{k}^{1}\right]$ and $\mathcal{M}_{\mathrm{loc}}:=\mathcal{M}\left[\mathbf{L}^{-1}\right]$. We denote by $\mathcal{M}[T]_{\text {loc }}$ the subring of $\mathcal{M}_{\text {loc }}[[T]]$ generated by $\mathcal{M}_{\text {loc }}[T]$ and the series $\left(1-\mathbf{L}^{a} T^{b}\right)^{-1}$ with $a$ in $\mathbf{Z}$ and $b$ in $\mathbf{N} \backslash\{0\}$.

Let $X$ be an algebraic variety over $k$. We denote by $\mathcal{L}(X)$ the scheme of germs of $\operatorname{arcs}$ on $X$. It is a scheme over $k$ and for any field extension $k \subset K$ there is a natural bijection

$$
\mathcal{L}(X)(K) \simeq \operatorname{Mor}_{k-\text { schemes }}(\operatorname{Spec} K[[t]], X),
$$

between the set of $K$-rational points of $\mathcal{L}(X)$ and the set of $K[[t]]$-rational points of $X$ (called the set of germs of arcs with coefficients in $K$ on $X$ ). More precisely, the scheme $\mathcal{L}(X)$ is defined as the projective limit $\mathcal{L}(X):=\lim \mathcal{L}_{n}(X)$, in the category of $k$-schemes, of the schemes $\mathcal{L}_{n}(X), n \in \mathbf{N}$, representing the functor

$$
R \mapsto \operatorname{Mor}_{k-\text { schemes }}\left(\operatorname{Spec} R[t] / t^{n+1} R[t], X\right),
$$

defined on the category of $k$-algebras. (Thus, for any $k$-algebra $R$, the set of $R$ rational points of $\mathcal{L}_{n}(X)$ is naturally identified with the set of $R[t] / t^{n+1} R[t]$-rational points of $X$.) The existence of $\mathcal{L}_{n}(X)$ is well known, cf. [B-L-R] p.276, and the projective limit exists since the transition morphisms are affine. We shall denote by $\pi_{n}$ the canonical morphism $\mathcal{L}(X) \rightarrow \mathcal{L}_{n}(X)$ corresponding to truncation of arcs. In the present paper, the schemes $\mathcal{L}(X)$ and $\mathcal{L}_{n}(X)$ will always be considered with their reduced structure. Note that the set-theoretical image $\pi_{n}(\mathcal{L}(X))$ is a constructible subset of $\mathcal{L}_{n}(X)$, as follows from a theorem of M. Greenberg [G], see (4.4) below. 
These constructible sets $\pi_{n}(\mathcal{L}(X))$ were first studied by J. Nash in [N], in relation with Hironaka's resolution of singularities. They are also considered in the papers $[\mathrm{L}-\mathrm{J}],[\mathrm{H}]$.

The following result is the first main result of the paper. It is an analogue of the rationality of the Poincaré series associated to the $p$-adic points on a variety proved in $[\mathrm{D} 1]$.

TheOREM 1.1. — Let $X$ be an algebraic variety over $k$. The power series

$$
P(T):=\sum_{n=0}^{\infty}\left[\pi_{n}(\mathcal{L}(X))\right] T^{n},
$$

considered as an element of $\mathcal{M}_{\text {loc }}[[T]]$, is rational and belongs to $\mathcal{M}[T]_{\text {loc }}$.

The proof of the theorem is given in section 5 and uses two main ingredients. The first one is a result of J. Pas $[\mathrm{P}]$ on quantifier elimination for semi-algebraic sets of power series in characteristic zero. The second one is M. Kontsevich's marvellous idea of motivic integration $[\mathrm{K}]$. More precisely, M. Kontsevich introduced the completion $\widehat{\mathcal{M}}$ of $\mathcal{M}_{\text {loc }}$ with respect to the filtration $F^{m} \mathcal{M}_{\text {loc }}$, where $F^{m} \mathcal{M}_{\text {loc }}$ is the subgroup of $\mathcal{M}_{\text {loc }}$ generated by $\left\{[S] \mathbf{L}^{-i} \mid i-\operatorname{dim} S \geq m\right\}$, and defined, for smooth $X$, a motivic integration on $\mathcal{L}(X)$ with values into $\widehat{\mathcal{M}}$. This is an analogue of classical $p$-adic integration. In the present paper we extend Kontsevich's construction to semi-algebraic subsets of $\mathcal{L}(X)$, with $X$ any pure dimensional algebraic variety over $k$, not necessarily smooth. For such an $X$, let $\mathbf{B}$ be the set of all semi-algebraic subsets of $\mathcal{L}(X)$. We construct in section 3 a canonical measure $\mu: \mathbf{B} \rightarrow \widehat{\mathcal{M}}$. This allows us to define integrals

$$
\int_{A} \mathbf{L}^{-\alpha} d \mu
$$

for $A$ in $\mathbf{B}$ and $\alpha: A \rightarrow \mathbf{Z} \cup\{+\infty\}$ a simple function which is bounded from below. (Semi-algebraic subsets of $\mathcal{L}(X)$ and simple functions are defined in section 2.) The properties of this motivic integration, together with resolution of singularities and the result of Pas, suffice to prove the rationality of the image of $P(T)$ in $\widehat{\mathcal{M}}[[T]]$. To prove the rationality of $P(T)$, considered as an element of $\mathcal{M}_{\text {loc }}[[T]]$, one needs a more refined argument based on Lemma 2.8 and the use of an obvious lifting $\tilde{\mu}(A)$ in $\mathcal{M}_{\text {loc }}$ of $\mu(A)$, when $A$ is a stable semi-algebraic subset of $\mathcal{L}(X)$ (a notion defined in section 2).

For an algebraic variety $X$, it is natural to consider its motivic volume $\mu(\mathcal{L}(X))$. In section 6 , we give explicit formulas for $\mu(\mathcal{L}(X))$ in terms of certain special resolutions of singularities of $X$ (which always exist). As a corollary we deduce that $\mu(\mathcal{L}(X))$ always belongs to a certain localization of the image of $\mathcal{M}_{\text {loc }}$ in $\widehat{\mathcal{M}}$ on which the Euler characteristic $\chi$ naturally extends with rational values. So we obtain a new invariant of $X$, the Euler characteristic $\chi(\mu(\mathcal{L}(X)))$, which is a rational number and coincides with the usual Euler characteristic of $X$ when $X$ is smooth. In section 7 , we prove that, when $X$ is of pure dimension $d$, the sequence $\left[\pi_{n}(\mathcal{L}(X))\right] \mathbf{L}^{-(n+1) d}$ converges to $\mu(\mathcal{L}(X))$ in $\widehat{\mathcal{M}}$. This result, which is an analogue of a $p$-adic result by J. Oesterlé [O], gives, in some sense, a precise meaning to Nash's guess one should 
consider the limit of the $\pi_{n}(\mathcal{L}(X))$ 's. We conclude the paper by some remarks on the Greenberg function in section 8 .

For related results concerning motivic Igusa functions, see [D-L].

\section{Semi-algebraic sets of power series}

(2.1) From now on we will denote by $\bar{k}$ a fixed algebraic closure of $k$, and by $\bar{k}((t))$ the fraction field of $\bar{k}[[t]]$, where $t$ is one variable. Let $x_{1}, \ldots, x_{m}$ be variables running over $\bar{k}((t))$ and let $\ell_{1}, \ldots, \ell_{r}$ be variables running over $\mathbf{Z}$. A semi-algebraic condition $\theta\left(x_{1}, \ldots, x_{m} ; \ell_{1}, \ldots, \ell_{r}\right)$ is a finite boolean combination of conditions of the form

$$
\begin{aligned}
\operatorname{ord}_{t} f_{1}\left(x_{1}, \ldots, x_{m}\right) & \geq \operatorname{ord}_{t} f_{2}\left(x_{1}, \ldots, x_{m}\right)+L\left(\ell_{1}, \ldots, \ell_{r}\right) \\
\operatorname{ord}_{t} f_{1}\left(x_{1}, \ldots, x_{m}\right) & \equiv L\left(\ell_{1}, \ldots, \ell_{r}\right) \bmod d
\end{aligned}
$$

and

$$
h\left(\overline{a c}\left(f_{1}\left(x_{1}, \ldots, x_{m}\right)\right), \ldots, \overline{a c}\left(f_{m^{\prime}}\left(x_{1}, \ldots, x_{m}\right)\right)\right)=0
$$

where $f_{i}$ and $h$ are polynomials over $k, L$ is a polynomial of degree $\leq 1$ over $\mathbf{Z}$, $d \in \mathbf{N}$, and $\overline{a c}(x)$ is the coefficient of lowest degree of $x$ in $\bar{k}((t))$ if $x \neq 0$, and is equal to 0 otherwise. Here we use the convention that $(+\infty)+\ell=+\infty$ and $+\infty \equiv \ell \bmod d$, for all $\ell \in \mathbf{Z}$. In particular the condition $f\left(x_{1}, \ldots, x_{m}\right)=0$ is a semi-algebraic condition, for $f$ a polynomial over $k$. A subset of $\bar{k}((t))^{m} \times \mathbf{Z}^{r}$ defined by a semi-algebraic condition is called semi-algebraic. One defines similarly semi-algebraic subsets of $K((t))^{m} \times \mathbf{Z}^{r}$ for $K$ an algebraically closed field containing $\bar{k}$.

A function $\alpha: \bar{k}((t))^{m} \times \mathbf{Z}^{n} \rightarrow \mathbf{Z}$ is called simple if its graph is semi-algebraic. An easy result of Presburger $[\mathrm{Pr}]$ implies that $\left(\exists \ell_{1} \in \mathbf{Z}\right) \theta\left(x_{1}, \ldots, x_{m} ; \ell_{1}, \ldots, \ell_{r}\right)$ is semi-algebraic when $\theta$ is a semi-algebraic condition.

We will use the following result on quantifier elimination due to J. Pas $[\mathrm{P}]$.

Theorem 2.1 (J. Pas $[\mathrm{P}])$. — If $\theta$ is a semi-algebraic condition, then

$$
\left(\exists x_{1} \in \bar{k}((t))\right) \theta\left(x_{1}, \ldots, x_{m} ; \ell_{1}, \ldots, \ell_{r}\right)
$$

is semi-algebraic. Furthermore, for any algebraically closed field $K$ containing $\bar{k}$,

$$
\left(\exists x_{1} \in K((t))\right) \theta\left(x_{1}, \ldots, x_{m} ; \ell_{1}, \ldots, \ell_{r}\right)
$$

is also semi-algebraic and may be defined by the same conditions (i.e. independently of $K)$.

Indeed, the first assertion follows from Theorem 4.1 in $[\mathrm{P}]$ together with Chevalley's constructibility theorem and the above mentioned result of Presburger; the second assertion follows directly from the remark at the begining of $\S 3$ of $[\mathrm{P}]$.

The theorem of Pas is a refinement of older quantifier elimination results of $\mathrm{Ax}$ and Kochen $[\mathrm{A}-\mathrm{K}]$, and of Delon [De]. 
(2.2) Let $X$ be an algebraic variety over $k$. For $x \in \mathcal{L}(X)$, we denote by $k_{x}$ the residue field of $x$ on $\mathcal{L}(X)$, and by $\tilde{x}$ the corresponding rational point $\tilde{x} \in \mathcal{L}(X)\left(k_{x}\right)=$ $X\left(k_{x}[[t]]\right)$. When there is no danger of confusion we will often write $x$ instead of $\tilde{x}$. A semi-algebraic family of semi-algebraic subsets (for $n=0$ a semi-algebraic subset) $A_{\ell}, \ell \in \mathbf{N}^{n}$, of $\mathcal{L}(X)$ is a family of subsets $A_{\ell}$ of $\mathcal{L}(X)$ such that there exists a covering of $X$ by affine Zariski open sets $U$ with

$$
A_{\ell} \cap \mathcal{L}(U)=\left\{x \in \mathcal{L}(U) \mid \theta\left(h_{1}(\tilde{x}), \ldots, h_{m}(\tilde{x}) ; \ell\right)\right\},
$$

where $h_{1}, \ldots, h_{m}$ are regular functions on $U$ and $\theta$ is a semi-algebraic condition. Here the $h_{i}$ 's and $\theta$ may depend on $U$ and $h_{i}(\tilde{x})$ belongs to $k_{x}[[t]]$.

Let $A$ be a semi-algebraic subset of $\mathcal{L}(X)$. A function $\alpha: A \times \mathbf{Z}^{n} \rightarrow \mathbf{Z} \cup$ $\{+\infty\}$ is called simple if the family of subsets $\left\{x \in \mathcal{L}(X) \mid \alpha\left(x, \ell_{1}, \ldots, \ell_{n}\right)=\ell_{n+1}\right\}$, $\left(\ell_{1}, \ldots, \ell_{n+1}\right) \in \mathbf{N}^{n+1}$, is a semi-algebraic family of semi-algebraic subsets of $\mathcal{L}(X)$.

A Presburger subset of $\mathbf{Z}^{r}$ is a subset defined by a semi-algebraic condition $\theta\left(\ell_{1}, \ldots, \ell_{r}\right)$ as in $(2.1)$ with $m=0$. A function $\alpha: \mathbf{Z}^{r} \rightarrow \mathbf{Z}$ is called a Presburger function if its graph is a Presburger subset of $\mathbf{Z}^{r+1}$.

If $f: X \rightarrow Y$ is a morphism of algebraic varieties over $k$ and $A$ is a semi-algebraic subset of $\mathcal{L}(X)$, then $f(A)$ is a semi-algebraic subset of $\mathcal{L}(Y)$, by Pas's Theorem.

We denote by $\pi_{n}$ the canonical morphism $\mathcal{L}(X) \rightarrow \mathcal{L}_{n}(X)$. If necessary we will use also the notation $\pi_{n, X}$. If $X$ is smooth, then $\pi_{n}$ is surjective by Hensel's Lemma. (2.3) The following basic result is a consequence of Pas's Theorem.

Proposition 2.3. - Let $X$ be an algebraic variety over $k$, and let $A$ be a semialgebraic subset of $\mathcal{L}(X)$. Then $\pi_{n}(A)$ is a constructible subset of $\mathcal{L}_{n}(X)$.

Proof. - We may assume $X=\mathbf{A}_{k}^{n}$. Let $s: \mathcal{L}_{n}(X) \rightarrow \mathcal{L}(X)$ be a section of $\pi_{n}: \mathcal{L}(X) \rightarrow \mathcal{L}_{n}(X)$ which maps $\mathcal{L}_{n}(X)(\bar{k})$ into $X(\bar{k}[t]) \subset X(\bar{k}[[t]])=\mathcal{L}(X)(\bar{k})$. We have $y \in \pi_{n}(A)$ if and only if $s(y) \in \pi_{n}^{-1} \pi_{n}(A)$. It is easy to verify that this implies the proposition because $\pi_{n}^{-1} \pi_{n}(A)$ is a semi-algebraic subset of $\mathcal{L}(X)$ by Theorem 2.1 .

(2.4) Let $A$ be a semi-algebraic subset of $\mathcal{L}(X)$. We call $A$ weakly stable at level $n \in \mathbf{N}$ if $A$ is a union of fibers of $\pi_{n}: \mathcal{L}(X) \rightarrow \mathcal{L}_{n}(X)$. We call $A$ weakly stable if it stable at some level $n$. Note that weakly stable semi-algebraic subsets form a boolean algebra.

LEMma 2.4. - For each $i \in \mathbf{N}$, let $A_{i}$ be a weakly stable semi-algebraic subset of $\mathcal{L}(X)$. Suppose that $A:=\bigcup_{i \in \mathbf{N}} A_{i}$ is semi-algebraic and weakly stable. Then $A$ equals the union of a finite number of the $A_{i}^{\prime} s$.

Proof. - We may assume $X$ is affine. By looking at the complements of the $A_{i}$ 's, it is enough to prove the following assertion. For each $i$ in $\mathbf{N}$, let $B_{i}$ be a weakly stable semi-algebraic subset of $\mathcal{L}(X)$, and suppose that the intersection of finitely many of the $B_{i}$ 's is always nonempty. Then $\bigcap_{i \in \mathbf{N}} B_{i}$ is nonempty. To prove this 
assertion note that each $B_{i}$ is a finite boolean combination of closed subschemes of $\mathcal{L}(X)$ whose ideals are finitely generated, since $B_{i}$ is weakly stable at some level $n_{i}$ and $\pi_{n_{i}}(B)$ is constructible. For any finite subset $\Sigma$ of $\mathbf{N}$ there exists a field $K_{\Sigma}$

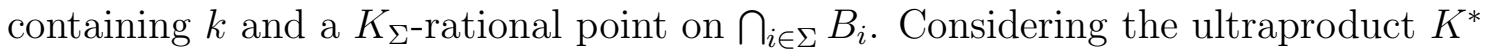
of the $K_{\Sigma}$ 's with respect to a suitable ultrafilter, we obtain a $K^{*}$-rational point in $\bigcap_{i \in \mathbf{N}} B_{i}$. (This kind of argument is very classical in model theory, see, e.g., page 172 of $[\mathrm{C}-\mathrm{K}]$.) This proves the assertion.

Remark. - The above lemma may also be deduced from Corollaire 7.2.7 of [G-D].

(2.5) Let $X, Y$ and $F$ be algebraic varieties over $k$, and let $A$, resp. $B$, be a constructible subset of $X$, resp. $Y$. We say that a map $\pi: A \rightarrow B$ is a piecewise trivial fibration with fiber $F$, if there exists a finite partition of $B$ in subsets $S$ which are locally closed in $Y$ such that $\pi^{-1}(S)$ is locally closed in $X$ and isomorphic, as a variety over $k$, to $S \times F$, with $\pi$ corresponding under the isomorphism to the projection $S \times F \rightarrow S$. We say that the map $\pi$ is a piecewise trivial fibration over some constructible subset $C$ of $B$, if the restriction of $\pi$ to $\pi^{-1}(C)$ is a piecewise trivial fibration onto $C$.

(2.6) For $X$ an algebraic variety over $k$ and $e$ in $\mathbf{N}$, we will use the notation

$$
\mathcal{L}^{(e)}(X):=\mathcal{L}(X) \backslash \pi_{e, X}^{-1}\left(\mathcal{L}_{e}\left(X_{\text {sing }}\right)\right),
$$

where $X_{\text {sing }}$ denotes the singular locus of $X$.

(2.7) Let $X$ be an algebraic variety over $k$ of pure dimension $d$ (in particular we assume that $X$ is non empty) and let $A$ be a semi-algebraic subset of $\mathcal{L}(X)$. We call $A$ stable at level $n \in \mathbf{N}$, if $A$ is weakly stable at level $n$ and $\pi_{m+1}(\mathcal{L}(X)) \rightarrow \pi_{m}(\mathcal{L}(X))$ is a piecewise trivial fibration over $\pi_{m}(A)$ with fiber $\mathbf{A}_{k}^{d}$ for all $m \geq n$.

We call $A$ stable if it stable at some level $n$. Note that the family of stable semi-algebraic subsets of $\mathcal{L}(X)$ is closed under taking finite intersections and finite unions. If $A$ is stable at level $n$, then $\left[\pi_{m}(A)\right]=\left[\pi_{n}(A)\right] \mathbf{L}^{(m-n) d}$ for all $m \geq n$. If $A$ is weakly stable and $A \cap \mathcal{L}\left(X_{\text {sing }}\right)=\emptyset$ (which is for instance the case when $X$ is smooth), then $A$ is stable. Indeed this follows from Lemma 2.4 and Lemma 4.1 below, because then $A$ is the union of the weakly stable subsets $A \cap \mathcal{L}^{(e)}(X)$.

(2.8) We say that a semi-algebraic family $A_{\ell}, \ell \in \mathbf{N}^{n}$, of semi-algebraic subsets of $\mathcal{L}(X)$, has a bounded representation if there exists a covering of $X$ by affine Zariski open sets $U$ such that on each $U$ the family is given by a semi-algebraic condition $\theta$ (cf. (2.2)) with, in the notation of (2.1), $\operatorname{ord}_{t} f_{i}$ bounded on $A_{\ell} \cap U$ for each fixed $\ell$.

Clearly, if the family $A_{\ell}$ has a bounded representation then each $A_{\ell}$ is weakly stable. The next lemma is essential for the proof of Theorem $5.1^{\prime}$ on which Theorem 1.1 is based.

Lemma 2.8. - Let $X$ be a quasi-projective algebraic variety over $k$ and let $A_{\ell}$, $\ell \in \mathbf{N}^{n}$, be a semi-algebraic family of semi-algebraic subsets of $\mathcal{L}(X)$. Assume that 
$A_{\ell}$ is weakly stable for each $\ell$. Then the family $A_{\ell}$ is a finite boolean combination of semi-algebraic families of semi-algebraic subsets of $\mathcal{L}(X)$ which have bounded representations.

Proof. - Because $X$ is quasi-projective we can work with a covering of $X$ by affine open sets $U_{i}, i \in I$, such that $U_{i} \backslash U_{j}$ is the locus in $U_{i}$ of a single regular function on $U_{i}$. Hence we may assume that $X$ is affine and that the family $A_{\ell}, \ell \in \mathbf{N}^{n}$, is given by a semi-algebraic condition as in (2.2) with $U=X$.

Let $f_{1}, \ldots, f_{r}$ be the regular functions on $X$ appearing in the conditions of the form 2.1 (i), (ii) and (iii) in the description of the semi-algebraic family $A_{\ell}$.

Assume that for $i=1,2, \ldots, e \leq r, \operatorname{ord}_{t} f_{i}$ is not bounded on $A_{\ell}$ for some $\ell$, possibly depending on $i$, and that $\operatorname{ord}_{t} f_{e+1}, \ldots, \operatorname{ord}_{t} f_{r}$ are bounded on $A_{\ell}$ by a function $\nu(\ell)$ of $\ell$. Our proof is by induction on $e$. We may assume that $A_{\ell}$ is weakly stable at level $\nu(\ell)$. Taking for $\nu(\ell)$ the smallest integer satisfying the above requirements, we see by Theorem 2.1 that $\nu: \mathbf{N}^{n} \rightarrow \mathbf{N}$ is a Presburger function.

By Greenberg's theorem [G], cf. (4.4) below, there exists a Presburger function $\alpha: \mathbf{N}^{n} \rightarrow \mathbf{N}$, with $\alpha(\ell) \geq \nu(\ell)$ for all $\ell \in \mathbf{N}^{n}$, such that, for all $x$ in $\mathcal{L}(X)$, if

$$
f_{1}(x) \equiv f_{2}(x) \equiv \cdots \equiv f_{e}(x) \equiv 0 \bmod t^{\alpha(\ell)+1}
$$

then there exists $x^{\prime}$ in $\mathcal{L}(X)$ with $x \equiv x^{\prime} \bmod t^{\nu(\ell)+1}$ and

$$
f_{1}\left(x^{\prime}\right)=f_{2}\left(x^{\prime}\right)=\cdots=f_{e}\left(x^{\prime}\right)=0 .
$$

Note that $A_{\ell}$ is the union of the following semi-algebraic subsets of $\mathcal{L}(X)$ which are weakly stable at level $\alpha(\ell)$ :

$$
\begin{aligned}
A_{\ell, 1} & :=A_{\ell} \cap\left\{x \in \mathcal{L}(X) \mid \operatorname{ord}_{t} f_{1}(x) \leq \alpha(\ell)\right\}, \\
& \vdots \\
A_{\ell, e} & :=A_{\ell} \cap\left\{x \in \mathcal{L}(X) \mid \operatorname{ord}_{t} f_{e}(x) \leq \alpha(\ell)\right\}, \\
B_{\ell} & :=A_{\ell} \cap\{x \in \mathcal{L}(X) \mid x \quad \text { satisfies } \quad(1)\} .
\end{aligned}
$$

The semi-algebraic families $A_{\ell, 1}, \ldots A_{\ell, e}, \ell \in \mathbf{N}^{n}$, are finite boolean combinations of semi-algebraic families which have bounded representations, because of the induction hypothesis. Thus it only remains to prove the lemma for the family $B_{\ell}$ instead of $A_{\ell}$. In the description of $A_{\ell}$ as a semi-algebraic family of semi-algebraic subsets of $\mathcal{L}(X)$, mentioned in the beginning of the proof, replace $f_{1}, f_{2}, \ldots, f_{e}$ by 0 and add the conditions $\operatorname{ord}_{t} f_{j}(x) \leq \nu(\ell)$ for $j=e+1, \ldots, r$. In this way we obtain a new semi-algebraic family of semi-algebraic subsets of $\mathcal{L}(X)$ which we denote by $A_{\ell}^{\prime}$, $\ell \in \mathbf{N}^{n}$. Clearly this family $A_{\ell}^{\prime}$ has a bounded representation and for each fixed $\ell$ the set $A_{\ell}^{\prime}$ is weakly stable at level $\nu(\ell)$.

If $x$ in $\mathcal{L}(X)$ satisfies $(1)$, then there exists $x^{\prime}$ in $\mathcal{L}(X)$, with $x \equiv x^{\prime} \bmod t^{\nu(\ell)+1}$, satisfying (2), and we have that $x \in A_{\ell}$ if and only if $x^{\prime} \in A_{\ell}$, since $A_{\ell}$ is weakly stable at level $\nu(\ell)$, which in turn is equivalent by (2) to $x^{\prime} \in A_{\ell}^{\prime}$ which, since $A_{\ell}^{\prime}$ is 
weakly stable at level $\nu(\ell)$, is verified if and only if $x \in A_{\ell}^{\prime}$. Thus

$$
\begin{aligned}
B_{\ell} & =A_{\ell}^{\prime} \cap\{x \in \mathcal{L}(X) \mid x \quad \text { satisfies }(1)\} \\
& =A_{\ell}^{\prime} \backslash \bigcup_{i=1, \ldots, e}\left\{x \in \mathcal{L}(X) \mid \operatorname{ord}_{t} f_{i}(x) \leq \alpha(\ell)\right\} .
\end{aligned}
$$

This proves the lemma, since $A_{\ell}^{\prime}$ and $\left\{x \in \mathcal{L}(X) \mid \operatorname{ord}_{t} f_{i}(x) \leq \alpha(\ell)\right\}$ have a bounded representation for each $i$.

\section{Motivic integration}

The basic idea of motivic integration goes back to M. Kontsevich [K]. We generalize here his idea to the much more general setting of semi-algebraic subsets of $\mathcal{L}(X)$ and simple functions on $\mathcal{L}(X)$, for $X$ an algebraic variety over $k$ which is not necessarily smooth.

(3.1) Let $X$ be an algebraic variety over $k$ of pure dimension $d$. Denote by $\mathbf{B}$ the set of all semi-algebraic subsets of $\mathcal{L}(X)$, and by $\mathbf{B}_{0}$ the set of all $A$ in $\mathbf{B}$ which are stable. Clearly there is a unique additive measure

$$
\tilde{\mu}: \mathbf{B}_{0} \longrightarrow \mathcal{M}_{\mathrm{loc}}
$$

satisfying

$$
\tilde{\mu}(A)=\left[\pi_{n}(A)\right] \mathbf{L}^{-(n+1) d}
$$

when $A$ is stable at level $n$. Sometimes we shall denote $\tilde{\mu}$ by $\tilde{\mu}_{\mathcal{L}(X)}$. Let $A$ be in $\mathbf{B}_{0}$ and let $\alpha: A \rightarrow \mathbf{Z}$ be a simple function all whose fibers are stable. Then, by Lemma 2.4, $|\alpha|$ is bounded, and we may define

$$
\int_{A} \mathbf{L}^{-\alpha} d \tilde{\mu}:=\sum_{n \in \mathbf{Z}} \mathbf{L}^{-n} \tilde{\mu}\left(\alpha^{-1}(n)\right),
$$

the sum at the right hand side being finite.

Next we want to extend the measure $\tilde{\mu}$ on $\mathbf{B}_{0}$ to a measure $\mu$ on $\mathbf{B}$. The key to achieving that is the following lemma which allows to partition any $A$ in $\mathbf{B}$ into stable subsets $A_{i}, i \in \mathbf{N}$, and a set of "measure zero". However, this leads to infinite sums, and for this reason $\mu$ will take values in the completion $\widehat{\mathcal{M}}$ of $\mathcal{M}_{\text {loc }}$, see (3.2) below.

LEMmA 3.1. - Let $X$ be an algebraic variety over $k$ of pure dimension $d$, and let $A$ be a semi-algebraic subset of $\mathcal{L}(X)$. There exists a closed subvariety $S$ of $X$, with $\operatorname{dim} S<\operatorname{dim} X$, and a semi-algebraic family $A_{i}, i \in \mathbf{N}$, of semi-algebraic subsets of $A$ such that $\mathcal{L}(S) \cap A$ and the $A_{i}$ 's form a partition of $A$, each $A_{i}$ is stable at some level $n_{i}$, and

$$
\lim _{i \rightarrow \infty}\left(\operatorname{dim} \pi_{n_{i}}\left(A_{i}\right)-\left(n_{i}+1\right) d\right)=-\infty .
$$

Moreover, if $\alpha: \mathcal{L}(X) \rightarrow \mathbf{Z}$ is a simple function, we can choose the partition such that $\alpha$ is constant on each $A_{i}$. 
Proof. - We may assume that $X$ is affine and irreducible and that $A$ is given by a semi-algebraic condition. Let $g$ be a nonzero regular function on $X$ which vanishes on the singular locus of $X$. Let $F$ be the product of $g$ and all the functions $f_{i}$ (assumed to be regular and not identically zero on $X$ ) appearing in the conditions of the form 2.1 (i), (ii) and (iii) in the description of the semi-algebraic set $A$. Then we can take $S$ to be the locus of $F=0$ and

$$
A_{i}=\left\{x \in A \backslash \mathcal{L}(S) \mid \operatorname{ord}_{t} F(x)=i\right\}
$$

Lemma 4.1 implies that $A_{i}$ is stable and (3.1.1) follows from Lemma 4.4. This proves the first assertion. The proof of the second assertion is quite similar.

(3.2) Let $S$ be an algebraic variety over $k$. We write $\operatorname{dim} S \leq n$ if all the irreducible components of $S$ have dimension $\leq n$. Similarly, for $M$ in $\mathcal{M}$, we write $\operatorname{dim} M \leq n$ if $M$ may be expressed as a linear combination of algebraic varieties with $\operatorname{dim} \leq n$. For $m$ in $\mathbf{Z}$, we denote by $F^{m} \mathcal{M}_{\text {loc }}$ the subgroup of $\mathcal{M}_{\text {loc }}$ generated by $\left\{[S] \mathbf{L}^{-i}\right.$ $i-\operatorname{dim} S \geq m\}$. This defines a decreasing filtration $F^{m}$ on $\mathcal{M}_{\text {loc }}$. We denote by $\widehat{\mathcal{M}}$ the completion of $\mathcal{M}_{\text {loc }}$ with respect to this filtration. We do not know whether or not the natural morphism $\mathcal{M}_{\text {loc }} \rightarrow \widehat{\mathcal{M}}$ is injective, but what is important for the applications in section 6 is the fact that the Euler characteristic and the Hodge polynomial of an algebraic variety $S$ only depend on the image of $[S]$ in $\widehat{\mathcal{M}}$, see $(6.1)$ below.

We denote by $\overline{\mathcal{M}}_{\text {loc }}$ the image of $\mathcal{M}_{\text {loc }}$ in $\widehat{\mathcal{M}}$, thus

$$
\overline{\mathcal{M}}_{\text {loc }}=\mathcal{M}_{\text {loc }} / \cap_{m} F^{m} \mathcal{M}_{\text {loc }}
$$

Definition-Proposition 3.2. - Let $X$ be an algebraic variety over $k$ of pure dimension $d$. Let $\mathbf{B}$ be the set of all semi-algebraic subsets of $\mathcal{L}(X)$. There exists a unique map $\mu: \mathbf{B} \rightarrow \widehat{\mathcal{M}}$ satisfying the following three properties.

(3.2.1) If $A \in \mathbf{B}$ is stable at level $n$, then $\mu(A)=\left[\pi_{n}(A)\right] \mathbf{L}^{-(n+1) d}$.

(3.2.2) If $A \in \mathbf{B}$ is contained in $\mathcal{L}(S)$ with $S$ a closed subvariety of $X$ with $\operatorname{dim} S<\operatorname{dim} X$, then $\mu(A)=0$.

(3.2.3) Let $A_{i}$ be in $\mathbf{B}$ for each $i$ in $\mathbf{N}$. Assume that the $A_{i}$ 's are mutually disjoint and that $A:=\bigcup_{i \in \mathbf{N}} A_{i}$ is semi-algebraic. Then $\sum_{i \in \mathbf{N}} \mu\left(A_{i}\right)$ converges in $\widehat{\mathcal{M}}$ to $\mu(A)$.

We call this unique map the motivic volume on $\mathcal{L}(X)$ and denote it by $\mu_{\mathcal{L}(X)}$ or $\mu$. Moreover we have

(3.2.4) If $A$ and $B$ are in $\mathbf{B}, A \subset B$, and if $\mu(B)$ belongs to the closure $F^{m}(\widehat{\mathcal{M}})$ of $F^{m} \mathcal{M}_{\text {loc }}$ in $\widehat{\mathcal{M}}$, then $\mu(A) \in F^{m}(\widehat{\mathcal{M}})$. 
Hence, for $A$ in $\mathbf{B}$ and $\alpha: A \rightarrow \mathbf{Z} \cup\{+\infty\}$ a simple function, we can define

$$
\int_{A} \mathbf{L}^{-\alpha} d \mu:=\sum_{n \in \mathbf{Z}} \mu\left(A \cap \alpha^{-1}(n)\right) \mathbf{L}^{-n}
$$

in $\widehat{\mathcal{M}}$, whenever the right hand side converges in $\widehat{\mathcal{M}}$, in which case we say that $\mathbf{L}^{-\alpha}$ is integrable on $A$. If the function $\alpha$ is bounded from below, then $\mathbf{L}^{-\alpha}$ is integrable on $A$, because of (3.2.4).

Proof. - The key ingredients in the proof are the lemmas 2.4, 3.1 and 4.3. The uniqueness of $\mu$ follows directly from Lemma 3.1 , so it only remains to prove the existence of a map $\mu: \mathbf{B} \rightarrow \widehat{\mathcal{M}}$ satisfying (3.2.1) up to (3.2.4).

Let $\mathbf{B}_{0}$ denote the set of all $A$ in $\mathbf{B}$ which are stable. Thus $\mathbf{B}_{0}$ is closed under finite unions and finite intersections. Clearly, there exists a map $\mu_{0}: \mathbf{B}_{0} \rightarrow \widehat{\mathcal{M}}$ satisfying (3.2.1) and (3.2.4) with $\mu$ and $\mathbf{B}$ replaced by $\mu_{0}$ and $\mathbf{B}_{0}$. Obviously $\mu_{0}$ is additive, hence Lemma 2.4 yields (3.2.3) with $\mu$ and $\mathbf{B}$ replaced by $\mu_{0}$ and $\mathbf{B}_{0}$. Next let $\mathbf{B}_{1}$ be the set of all $A$ in $\mathbf{B}$ which can be written as $A=\bigcup_{i \in \mathbf{N}} A_{i}$ with the $A_{i}$ 's in $\mathbf{B}_{0}$ mutually disjoint and $\lim _{i \rightarrow \infty} \mu_{0}\left(A_{i}\right)=0$. For $A$ in $\mathbf{B}_{1}$ we set $\mu_{1}(A)=\sum_{i=0}^{\infty} \mu_{0}\left(A_{i}\right)$. This is independent of the choice of the $A_{i}$ 's. Indeed, suppose that also $A=\bigcup_{i \in \mathbf{N}} A_{i}^{\prime}$ with the $A_{i}^{\prime}$ 's in $\mathbf{B}_{0}$ mutually disjoint and $\lim _{i \rightarrow \infty} \mu_{0}\left(A_{i}^{\prime}\right)=0$. Then

$$
\begin{aligned}
\sum_{i=0}^{\infty} \mu_{0}\left(A_{i}\right) & =\sum_{i=0}^{\infty} \mu_{0}\left(\bigcup_{j \in \mathbf{N}}\left(A_{i} \cap A_{j}^{\prime}\right)\right)=\sum_{i=0}^{\infty} \sum_{j=0}^{\infty} \mu_{0}\left(A_{i} \cap A_{j}^{\prime}\right) \\
& =\sum_{j=0}^{\infty} \sum_{i=0}^{\infty} \mu_{0}\left(A_{i} \cap A_{j}^{\prime}\right)=\sum_{j=0}^{\infty} \mu_{0}\left(A_{j}^{\prime}\right)
\end{aligned}
$$

because (3.2.3) and (3.2.4) hold for $\mu$ and $\mathbf{B}$ replaced by $\mu_{0}$ and $\mathbf{B}_{0}$. One verifies that (3.2.1) and (3.2.4) are true for $\mu$ and $\mathbf{B}$ replaced by $\mu_{1}$ and $\mathbf{B}_{1}$. From Lemma 4.3 one easily deduces the following

(3.2.5) If $S$ is a closed subvariety of $X$ with $\operatorname{dim} S<\operatorname{dim} X$ and if $A$ belongs to $\mathbf{B}_{1}$, then $A \backslash \mathcal{L}(S)$ belongs also to $\mathbf{B}_{1}$ and $\mu_{1}(A \backslash \mathcal{L}(S))=\mu_{1}(A)$.

Indeed, we may assume $A$ belongs to $\mathbf{B}_{0}$ and consider the following partitions by elements of $\mathbf{B}_{0}$ :

$$
\begin{gathered}
A \backslash \mathcal{L}(S)=\left(A \backslash \pi_{m}^{-1} \pi_{m}(\mathcal{L}(S))\right) \cup \bigcup_{n \geq m}\left(\left(\pi_{n}^{-1} \pi_{n}(\mathcal{L}(S)) \backslash \pi_{n+1}^{-1} \pi_{n+1}(\mathcal{L}(S))\right) \cap A\right), \\
A=\left(A \backslash \pi_{m}^{-1} \pi_{m}(\mathcal{L}(S))\right) \cup\left(\pi_{m}^{-1} \pi_{m}(\mathcal{L}(S)) \cap A\right),
\end{gathered}
$$

for $m \in \mathbf{N}$ large enough.

Next let $A$ be any element of B. Then, by Lemma 3.1, there exists a closed subvariety $S$ of $X$ with $\operatorname{dim} S<\operatorname{dim} X$ such that $A \backslash \mathcal{L}(S)$ belongs to $\mathbf{B}_{1}$. Define $\mu$ by $\mu(A)=\mu_{1}(A \backslash \mathcal{L}(S))$. By (3.2.5), this definition is independent of the choice of 
$S$. Indeed, if $S^{\prime}$ is another closed subvariety of $X$ with $\operatorname{dim} S^{\prime}<\operatorname{dim} X$ such that $A \backslash \mathcal{L}\left(S^{\prime}\right)$ belongs to $\mathbf{B}_{1}$, then

$$
\begin{aligned}
\mu_{1}\left(A \backslash \mathcal{L}\left(S^{\prime}\right)\right) & =\mu_{1}\left(\left(A \backslash \mathcal{L}\left(S^{\prime}\right)\right) \backslash \mathcal{L}(S)\right)=\mu_{1}\left((A \backslash \mathcal{L}(S)) \backslash \mathcal{L}\left(S^{\prime}\right)\right) \\
& =\mu_{1}(A \backslash \mathcal{L}(S))
\end{aligned}
$$

Clearly (3.2.1), (3.2.2) and (3.2.4) are satisfied and $\mu$ is additive on finite disjoint unions. It remains to prove (3.2.3). Let $A$ and the $A_{i}$ 's be elements of $\mathbf{B}$ as in (3.2.3) and let $m$ be in $\mathbf{N}$. By an argument entirely similar to the proof of Lemma 3.1, there exists weakly stable $A^{\prime}$ and $A_{i}^{\prime}$ 's in $\mathbf{B}$ such that $A \subset A^{\prime}, A_{i} \subset A_{i}^{\prime}$ and $\mu(A)-\mu\left(A^{\prime}\right)$ and $\mu\left(A_{i}\right)-\mu\left(A_{i}^{\prime}\right)$ belong to $F^{m} \widehat{\mathcal{M}}$. Moreover, replacing $A_{i}$ by $A_{i} \cup\left(A^{\prime} \backslash A\right)$ and $A_{i}^{\prime}$ by $A_{i}^{\prime} \cap A^{\prime}$, we may assume $A_{i} \cup\left(A^{\prime} \backslash A\right) \subset A_{i}^{\prime} \subset A^{\prime}$. Hence $A^{\prime}=\bigcup_{i \in \mathbf{N}} A_{i}^{\prime}$, and by Lemma 2.4, $A^{\prime}$ is the union of a finite number of the sets $A_{i}^{\prime}$, thus $A^{\prime}=\bigcup_{i=1, \ldots, e} A_{i}^{\prime}$ whenever $e$ is large enough. Since

$$
A^{\prime}=\left(\bigcup_{i=1, \ldots, e} A_{i}\right) \cup\left(\bigcup_{i=1, \ldots, e}\left(A_{i}^{\prime} \backslash A_{i}\right)\right)
$$

we get

$$
\mu(A) \equiv \mu\left(A^{\prime}\right) \equiv \sum_{i=1}^{e} \mu\left(A_{i}\right) \quad \bmod \quad F^{m} \widehat{\mathcal{M}}
$$

Because this holds for all $m$ in $\mathbf{N}$, we obtain (3.2.3).

(3.3) Let $X$ be an algebraic variety over $k$ of dimension $d$, and let $\mathcal{I}$ be a coherent sheaf of ideals on $X$. We denote by $\operatorname{ord}_{t} \mathcal{I}$ the function $\operatorname{ord}_{t} \mathcal{I}: \mathcal{L}(X) \rightarrow \mathbf{N} \cup\{+\infty\}$ given by $\varphi \mapsto \min _{g} \operatorname{ord}_{t} g(\tilde{\varphi})$, where the minimum is taken over all $g$ in the stalk $\mathcal{I}_{\pi_{0}(\varphi)}$ of $\mathcal{I}$ at $\pi_{0}(\varphi)$. Note that $\operatorname{ord}_{t} \mathcal{I}$ is a simple function. Let $\Omega_{X}^{1}$ be the sheaf of differentials on $X$ and let $\Omega_{X}^{d}$ be the $d$-th exterior power of $\Omega_{X}^{1}$. If $X$ is smooth and $\mathcal{F}$ is a coherent sheaf on $X$ together with a natural morphism $\iota: \mathcal{F} \rightarrow \Omega_{X}^{d}$, we denote by $\mathcal{I}(\mathcal{F})$ the sheaf of ideals on $X$ which is locally generated by functions $\iota(\omega) / d x$ with $\omega$ a local section of $\mathcal{F}$ and $d x$ a local volume form on $X$. Denote by $\operatorname{ord}_{t} \mathcal{F}$ the simple function $\operatorname{ord}_{t} \mathcal{I}(\mathcal{F})$.

We have the following change of variables formula for birational morphisms, which generalizes the one in $[\mathrm{K}]$.

Lemma 3.3. - Let $X$ and $Y$ be algebraic varieties over $k$, of pure dimension $d$. Assume that $Y$ is smooth. Let $h: Y \rightarrow X$ be a proper birational morphism, $A$ be a semi-algebraic subset of $\mathcal{L}(X)$ and let $\alpha: A \rightarrow \mathbf{N}$ be a simple function. Then

$$
\int_{A} \mathbf{L}^{-\alpha} d \mu=\int_{h^{-1}(A)} \mathbf{L}^{-\alpha \circ h-\operatorname{ord}_{t} h^{*}\left(\Omega_{X}^{d}\right)} d \mu .
$$

Moreover assume that $A \cap \mathcal{L}(h(E))=\emptyset$, where $E$ is the exceptional locus of $h$, and that $A$ and the fibers of $\alpha$ are weakly stable (and hence stable). Then $h^{-1}(A)$ and the fibers of $\alpha \circ h+\operatorname{ord}_{t} h^{*}\left(\Omega_{X}^{d}\right)$ on $h^{-1}(A)$ are stable and the above formula also holds for $\mu$ replaced by $\tilde{\mu}$. 
Proof. - The first assertion follows directly from Lemma 3.1 and Lemma 3.4 below. Now assume that $A \cap \mathcal{L}(h(E))=\emptyset$ and that $A$ and the fibers of $\alpha$ are weakly stable. Since $X_{\text {sing }} \subset h(E)$, we have $A \cap \mathcal{L}\left(X_{\text {sing }}\right)=\emptyset$. Hence $A$ and the fibers of $\alpha$ are stable by (2.7). Moreover $h^{-1}(A)$ is stable, because $Y$ is smooth. Note that $\operatorname{ord}_{t} h^{*}\left(\Omega_{X}^{d}\right)$ does not take the value $+\infty$ on $h^{-1}(A)$, since $h^{-1}(A) \cap \mathcal{L}(E)=\emptyset$, and that the fibers of $\operatorname{ord}_{t} h^{*}\left(\Omega_{X}^{d}\right)$ on $h^{-1}(A)$ are weakly stable, hence stable. Thus, by (3.1), $\alpha \circ h$ and $\operatorname{ord}_{t} h^{*}\left(\Omega_{X}^{d}\right)$ are bounded on $h^{-1}(A)$. We conclude that the fibers of $\alpha \circ h+\operatorname{ord}_{t} h^{*}\left(\Omega_{X}^{d}\right)$ on $h^{-1}(A)$ are stable, because they are finite unions of intersections of a fiber of $\alpha \circ h$ with a fiber of $\operatorname{ord}_{t} h^{*}\left(\Omega_{X}^{d}\right)$. Note also that $A \subset \mathcal{L}^{\left(e^{\prime}\right)}(X)$ for some $e^{\prime}$ in $\mathbf{N}$, by Lemma 2.4. The last assertion of Lemma 3.3 follows now directly from Lemma 3.4 below.

LEMMA 3.4. - Let $X$ and $Y$ be algebraic varieties over $k$, of pure dimension d and let $h: Y \rightarrow X$ be a birational morphism. Assume that $Y$ is smooth. For $e$ and $e^{\prime}$ in $\mathbf{N}$, let $\Delta_{e, e^{\prime}}$ be the semi-algebraic subset of $\mathcal{L}(Y)$ defined by

$$
\Delta_{e, e^{\prime}}:=\left\{\varphi \in \mathcal{L}(Y) \mid\left(\operatorname{ord}_{t} h^{*}\left(\Omega_{X}^{d}\right)\right)(\varphi)=e \text { and } h(\varphi) \in \mathcal{L}^{\left(e^{\prime}\right)}(X)\right\}
$$

where $\mathcal{L}^{\left(e^{\prime}\right)}(X)$ is defined as in 2.6. For $n$ in $\mathbf{N}$, let $h_{n *}: \mathcal{L}_{n}(Y) \rightarrow \mathcal{L}_{n}(X)$ be the morphism induced by $h$, and let $\Delta_{e, e^{\prime}, n}$ be the image of $\Delta_{e, e^{\prime}}$ in $\mathcal{L}_{n}(Y)$. There exists $c$ in $\mathbf{N} \backslash\{0\}$, such that, for all $e, e^{\prime}, n$ in $\mathbf{N}$ with $n \geq 2 e, n \geq e+c e^{\prime}$, the following holds.

(a) The set $\Delta_{e, e^{\prime}, n}$ is a union of fibers of $h_{n *}$.

(b) The restriction of $h_{n *}$ to $\Delta_{e, e^{\prime}, n}$ is a piecewise trivial fibration with fiber $\mathbf{A}_{k}^{e}$ onto its image.

Proof. - Because $Y$ is smooth, the canonical morphism $\mathcal{L}(Y) \rightarrow \mathcal{L}_{n}(Y)$ is surjective. Consider the following assertion :

$\left(\mathrm{a}^{\prime}\right) \quad h_{n *}^{-1}\left(h_{n *}(\bar{\varphi})\right) \subset\left\{\bar{y} \in \mathcal{L}_{n}(Y) \mid \bar{\varphi} \equiv \bar{y} \quad \bmod \mathcal{L}_{n-e}(Y)\right\} \quad$ for all $\quad \bar{\varphi} \in \Delta_{e, e^{\prime}, n}$,

where $\bar{\varphi} \equiv \bar{y} \bmod \mathcal{L}_{n-e}(Y)$ means that $\bar{\varphi}$ and $\bar{y}$ have the same image in $\mathcal{L}_{n-e}(Y)$. Since $n-e \geq e, e^{\prime}$, the right hand side of $\left(\mathrm{a}^{\prime}\right)$ is contained in $\Delta_{e, e^{\prime}, n}$, and assertion $\left(\mathrm{a}^{\prime}\right)$ implies assertion (a). Because $h$ is birational, $Y$ is smooth, $X$ is smooth at each point of $h\left(\Delta_{e, e^{\prime}}\right)$, and the ideal sheaf $\mathcal{I}\left(h^{*}\left(\Omega_{X}^{d}\right)\right)$ does not vanish at any point of $\Delta_{e, e^{\prime}}$, we have that $h(y) \neq h\left(y^{\prime}\right)$ whenever $y \neq y^{\prime}, y \in \Delta_{e, e^{\prime}}, y^{\prime} \in \mathcal{L}(Y)$. Hence assertion $\left(\mathrm{a}^{\prime}\right)$ is implied by the following assertion :

$\left(\mathrm{a}^{\prime \prime}\right) \quad$ For all $\varphi \in \Delta_{e, e^{\prime}}, x \in \mathcal{L}(X)$, with $h(\varphi) \equiv x \bmod \mathcal{L}_{n}(X)$, there exists $y \in \mathcal{L}(Y)$ with $h(y)=x$ and $\varphi \equiv y \quad \bmod \mathcal{L}_{n-e}(Y)$.

Thus, we have only to prove $\left(\mathrm{a}^{\prime \prime}\right)$ and (b). For this we may assume that $X$ and $Y$ are affine. Moreover we may assume that $Y \subset \mathbf{A}_{k}^{M}$ and that the first $d$ coordinates $y_{1}, y_{2}, \ldots, y_{d}$ on $\mathbf{A}_{k}^{M}$ induce an étale map $Y \rightarrow \mathbf{A}_{k}^{d}$. Then, by Lemma 4.2 (with $n=e=0$ ), the natural map $\mathcal{L}_{n}(Y) \rightarrow Y \times_{\mathbf{A}_{k}^{d}} \mathcal{L}_{n}\left(\mathbf{A}_{k}^{d}\right)$ is an isomorphism. For ease of 
notation we will assume that $Y=\mathbf{A}_{k}^{d}$. The general case can be proved in the same way, identifying $\mathcal{L}(Y)$ with $\mathcal{L}\left(\mathbf{A}_{k}^{d}\right)$ on each fixed fiber of $\mathcal{L}(Y) \rightarrow Y$, and taking for $\mathcal{J}_{h}$ below the jacobian matrix of $h$ with respect to the system of local coordinates $y_{1}, y_{2}, \ldots, y_{d}$ on $Y$.

Let us first prove $\left(\mathrm{a}^{\prime \prime}\right)$ and (b) in the special case where also $X=\mathbf{A}_{k}^{d}$. Let $\varphi$ be in $\Delta_{e, e^{\prime}}(\bar{k}) \subset \mathcal{L}(Y)(\bar{k})=Y(\bar{k}[[t]])$. Denote by $\mathcal{J}_{h}$ the jacobian matrix of $h$. For $\left(\mathrm{a}^{\prime \prime}\right)$ we have to prove that for all $v$ in $\bar{k}[[t]]^{d}$ there exists $u$ in $\bar{k}[[t]]^{d}$ such that

$$
h\left(\varphi+t^{n+1-e} u\right)=h(\varphi)+t^{n+1} v .
$$

By Taylor expansion, (1) is equivalent to

$$
\left.t^{-e} \mathcal{J}_{h}(\varphi) u+t \text { (higher order terms in } u\right)=v \text {. }
$$

The existence of a solution $u$ follows from Hensel's Lemma, since the inverse of $t^{-e} \mathcal{J}_{h}(\varphi)$ has entries in $\bar{k}[[t]]$, because $\operatorname{ord}_{t} \operatorname{det} \mathcal{J}_{h}(\varphi)=e$. This proves $\left(\mathrm{a}^{\prime \prime}\right)$, hence $\left(\mathrm{a}^{\prime}\right)$ and $(\mathrm{a})$, when $X=\mathbf{A}_{k}^{d}$.

Let us prove now assertion (b) assuming $X=\mathbf{A}_{k}^{d}$. Let the morphism $s$ : $\mathcal{L}_{n}(X) \rightarrow \mathcal{L}(X)$ be a section of the projection $\pi_{n}: \mathcal{L}(X) \rightarrow \mathcal{L}_{n}(X)$. For $\bar{x}$ in $h_{n *}\left(\Delta_{e, e^{\prime}, n}(\bar{k})\right), s(\bar{x})$ belongs to $h\left(\Delta_{e, e^{\prime}}\right)$, because of $(1)$, and hence $h^{-1}$ is regular at $s(\bar{x})$. The mapping $\theta: \bar{x} \mapsto h^{-1}(s(\bar{x}))$ from $h_{n *}\left(\Delta_{e, e^{\prime}, n}\right)$ to $\Delta_{e, e^{\prime}}$ is a piecewise morphism, meaning there exists a finite partition of the domain of $\theta$ into locally closed subvarieties of $\mathcal{L}_{n}(X)$, such that the restriction of $\theta$ to any subvariety is a morphism of schemes. For $\bar{x}$ in $h_{n *}\left(\Delta_{e, e^{\prime}, n}(\bar{k})\right)$, we deduce from $\left(\mathrm{a}^{\prime}\right)$ and $(2)$ that

$$
h_{n *}^{-1}(\bar{x})=\left\{\theta(\bar{x})+t^{n+1-e} u \bmod t^{n+1} \mid u \in \bar{k}[[t]]^{d} \text { and }\left(\mathcal{J}_{h}(\theta(\bar{x}))\right) u \equiv 0 \bmod t^{e}\right\}
$$

Thus we see that the fiber $h_{n *}^{-1}(\bar{x})$ can be identified with a linear subspace of $\left\{u \bmod t^{e} \mid u \in \bar{k}[[t]]^{d}\right\} \simeq \mathbf{A}_{k}^{d e}$ given by linear equations whose coefficients in $\bar{k}[[t]]$ are functions of $\bar{x}$ which are piecewise morphisms on $h_{n *}\left(\Delta_{e, e^{\prime}, n}\right) \subset \mathcal{L}_{n}(X)$. Moreover, for a fixed $\bar{x}$ in $h_{n *}\left(\Delta_{e, e^{\prime}, n}(\bar{k})\right)$, the jacobian matrix $\mathcal{J}_{h}(\theta(\bar{x}))$ is equivalent over $\bar{k}[[t]]$ to a diagonal matrix with diagonal elements $t^{e_{1}}, \ldots, t^{e_{d}}$, with $e_{1}, \ldots, e_{d}$ in $\mathbf{N}$, and $e_{1}+\cdots+e_{d}=e$. Together with (3), this gives an isomorphism

$$
h_{n *}^{-1}(\bar{x}) \simeq \mathbf{A}_{\bar{k}}^{e},
$$

and it is now easy to verify that $h_{\left.n *\right|_{e, e^{\prime}, n}}$ is a piecewise trivial fibration onto its image. This proves (b) when $X=\mathbf{A}_{k}^{d}$.

We now turn to the case where $X=\operatorname{Spec}\left(k\left[x_{1}, \ldots, x_{N}\right] / I\right) \subset \mathbf{A}_{k}^{N}$ and $Y=\mathbf{A}_{k}^{d}$. Denote again by $\mathcal{J}_{h}$ the jacobian matrix of $h: Y \rightarrow X \hookrightarrow \mathbf{A}_{k}^{N}$. By the argument at the beginning of the proof of Lemma 4.1, we see that there exists $c$ in $\mathbf{N} \backslash\{0\}$, such that, for any $e, e^{\prime}$ in $\mathbf{N}$, the set $h\left(\Delta_{e, e^{\prime}}\right)$ is covered by a finite number of semialgebraic subsets $A$ of $\mathcal{L}(X)$, which are weakly stable at level $c e^{\prime}$, such that, for each such $A$, the following holds : On $A$ the variety $X$ is a "complete intersection", meaning that

$$
\mathcal{L}(X) \cap A=\mathcal{L}\left(\operatorname{Spec}\left(k\left[x_{1}, \ldots, x_{N}\right] /\left(f_{1}, \ldots, f_{N-d}\right)\right)\right) \cap A,
$$


for suitable $f_{1}, \ldots, f_{N-d} \in I$ (which may depend on $A$ ), and moreover, for each $x$ in $A$, there exists an $N-d$ by $N-d$ minor $\delta^{\prime}$ of the matrix $\Delta:=\frac{\partial\left(f_{1}, \ldots, f_{N-d}\right)}{\partial\left(x_{1}, \ldots, x_{N}\right)}$ satisfying $\operatorname{ord}_{t} \delta^{\prime}(\tilde{x}) \leq c e^{\prime}$. Denote by $\delta$ the minor of $\Delta$ formed by the first $N-d$ columns. Up to shrinking $A$ and renumbering the coordinates, we may assume there exists $e^{\prime \prime}$ in $\mathbf{N}$, with $e^{\prime \prime} \leq c e^{\prime}$, such that, for all $x$ in $A$,

$$
e^{\prime \prime}=\operatorname{ord}_{t} \delta(\tilde{x}) \leq \operatorname{ord}_{t} \delta^{\prime}(\tilde{x})
$$

for all $N-d$ by $N-d$ minors $\delta^{\prime}$ of $\Delta$. It is enough to prove (a") and (b) for $\Delta_{e, e^{\prime}}$ replaced by $\Delta_{e, e^{\prime}} \cap h^{-1}(A)$. From now on let $\varphi$ be in $\Delta_{e, e^{\prime}}(\bar{k}) \cap h^{-1}(A)$ and assume $n \geq 2 e, n \geq e+c e^{\prime}$. Since the product of the jacobian matrices $\Delta(h(\varphi))$ and $\mathcal{J}_{h}(\varphi)$ yields zero, one checks that the $d$ by $d$ minor of $\mathcal{J}_{h}(\varphi)$ formed by the last $d$ rows has minimal ord or $_{t}$ equal to $e$, among all $d$ by $d$ minors of $\mathcal{J}_{h}(\varphi)$. Indeed, the columns of $\mathcal{J}_{h}(\varphi)$ are solutions of the homogeneous linear system of equations with matrix $\Delta(h(\varphi))$, and the first $N-d$ components of any solution are fixed $\bar{k}[[t]]$ linear combinations of the last $d$ components by Cramer's rule and (5). Thus the first $N-d$ rows of $\mathcal{J}_{h}(\varphi)$ are $\bar{k}[[t]]$-linear combinations of the last $d$ rows.

Let $p: X \rightarrow \mathbf{A}_{k}^{d}$ denote the projection on the last $d$ coordinates $\left(x_{1}, \ldots, x_{N}\right) \mapsto$ $\left(x_{N-d+1}, \ldots, x_{N}\right)$. Denote by $\mathcal{J}_{p \circ h}$ the jacobian matrix of $p \circ h$, i.e. $\mathcal{J}_{\text {poh }}$ consists in the last $d$ rows of the matrix $\mathcal{J}_{h}$. Thus

$$
e=\left(\operatorname{ord}_{t} h^{*}\left(\Omega_{X}^{d}\right)\right)(\varphi)=\operatorname{ord}_{t} \operatorname{det} \mathcal{J}_{p \circ h}(\varphi)
$$

To prove $\left(\mathrm{a}^{\prime \prime}\right)$ we have to show that for all $v$ in $\bar{k}[[t]]^{N}$, satisfying

$$
h(\varphi)+t^{n+1} v \in \mathcal{L}(X)(\bar{k}) \subset \bar{k}[[t]]^{N},
$$

there exists $u$ in $\bar{k}[[t]]^{d}$ such that

$$
h\left(\varphi+t^{n+1-e} u\right)=h(\varphi)+t^{n+1} v
$$

It follows from Lemma 4.2 (with $n$, $e$ replaced by $n-e, e^{\prime \prime}$ ) (or alternatively by a direct argument using Taylor expansion) that $\left(1^{\prime}\right)$ is equivalent to

$$
p \circ h\left(\varphi+t^{n+1-e} u\right)=p \circ h(\varphi)+t^{n+1} p(v) .
$$

The proof of $\left(\mathrm{a}^{\prime \prime}\right)$ and (b) proceeds now in the same way as in the special case $X=\mathbf{A}_{k}^{d}$ treated above from (1) to (4). We only have to replace $\mathcal{J}_{h}$ by $\mathcal{J}_{p \circ h}, v$ by $p(v),(1)$ by $\left(1^{\prime}\right), \Delta_{e, e^{\prime}}$ by $\Delta_{e, e^{\prime}} \cap h^{-1}(A), \Delta_{e, e^{\prime}, n}$ by the image of $\Delta_{e, e^{\prime}} \cap h^{-1}(A)$ in $\mathcal{L}_{n}(Y)$, and to take for $s: \mathcal{L}_{n}(X) \rightarrow \mathcal{L}(X)$ a section of the projection $\pi_{n}: \mathcal{L}(X) \rightarrow \mathcal{L}_{n}(X)$, whose restriction to $\pi_{n}(A)$ is a piecewise morphism. The existence of such a section $s$ is insured by Lemma 4.2. The essential point is that (3) remains valid when one replaces $\mathcal{J}_{h}$ by $\mathcal{J}_{p \circ h}$, because the first $N-d$ rows of $\mathcal{J}_{h}(\varphi)$ are $\bar{k}[[t]]$-linear combinations of the last $d$ rows. With these modifications, the argument from (1) to (4) remains valid in the present situation. This ends the proof of Lemma 3.4. 
Remark. - When $X$ is smooth and $\mathcal{I}$ is the ideal sheaf of an effective divisor $D$ on $X$, the motivic integral $\int_{\mathcal{L}(X)} \mathbf{L}^{-\operatorname{ord}_{t} \mathcal{I}} d \mu$ was first introduced by Kontsevich $[\mathrm{K}]$ and denoted by him $\left[\int_{X} e^{D}\right]$.

(3.5) In this subsection we consider a generalization which will not be used in the present paper. Let $X$ be an algebraic variety over $k$ of pure dimension $d$, and let $\mathcal{F}$ be a coherent sheaf on $X$ together with a natural morphism $\iota: \mathcal{F} \rightarrow \Omega_{X}^{d}$. We denote by $\operatorname{ord}_{t}(\mathcal{F})$ the simple function from $\mathcal{L}(X) \backslash \mathcal{L}\left(X_{\text {sing }}\right)$ to $\mathbf{N} \cup\{+\infty\}$ defined by

$$
\left.\operatorname{cord}_{t} \mathcal{F}\right)(\varphi)=\min _{\omega_{1} \in \mathcal{F}_{\pi_{0}}(\varphi)} \max _{\omega_{2} \in\left(\Omega_{X}^{d}\right)_{\pi_{0}(\varphi)}} \operatorname{ord}_{t} \frac{\iota\left(\omega_{1}\right)}{\omega_{2}}(\tilde{\varphi}),
$$

for any $\varphi$ in $\mathcal{L}(X) \backslash \mathcal{L}\left(X_{\text {sing }}\right)$. (Here $X_{\text {sing }}$ denotes the singular locus of $X$.) This definition of $\operatorname{ord}_{t}(\mathcal{F})$ coincides with the one given in (3.3) when $X$ is smooth. By (3.2), the map

$$
\mathbf{B} \rightarrow \widehat{\mathcal{M}}: \quad A \mapsto \int_{A \backslash \mathcal{L}\left(X_{\text {sing }}\right)} \mathbf{L}^{-\operatorname{ord}_{t} \mathcal{F}} d \mu_{\mathcal{L}(X)}
$$

is a $\sigma$-additive measure on $\mathcal{L}(X)$ which we will denote by $\mu_{\mathcal{F}}$. Note that $\mu_{\Omega_{X}^{d}}=\mu_{\mathcal{L}(X)}$. Let $A$ be a semi-algebraic subset of $\mathcal{L}(X)$ and let $\alpha: A \rightarrow \mathbf{N} \cup\{+\infty\}$ be a simple function. We define the motivic integral $\int_{A} \mathbf{L}^{-\alpha} d \mu_{\mathcal{F}}$ as in (3.2), but with $\mu$ replaced by $\mu_{\mathcal{F}}$. If $Y$ is any algebraic variety over $k$ of pure dimension $d$ and $h: Y \rightarrow X$ is a proper birational morphism, then

$$
\int_{h^{-1}(A)} \mathbf{L}^{-\alpha \circ h} d \mu_{h^{*}(\mathcal{F})}=\int_{A} \mathbf{L}^{-\alpha} d \mu_{\mathcal{F}}
$$

Indeed, this follows by considering a resolution of $Y$ and applying Lemma 3.3 twice.

\section{Some lemmas}

In this section we prove some lemmas which were already used in sections 2 and 3 and which will be used again in section 7 .

Lemma 4.1. - Let $X$ be an algebraic variety over $k$ of pure dimension $d$, and assume the notation of 2.6. There exists $c$ in $\mathbf{N} \backslash\{0\}$ such that, for all $e, n$ in $\mathbf{N}$ with $n \geq$ ce, the following holds.

(a) The map

$$
\theta_{n}: \pi_{n+1}(\mathcal{L}(X)) \rightarrow \pi_{n}(\mathcal{L}(X))
$$

is a piecewise trivial fibration over $\pi_{n}\left(\mathcal{L}^{(e)}(X)\right)$ with fiber $\mathbf{A}_{k}^{d}$.

(b) Moreover

$$
\left[\pi_{n}\left(\mathcal{L}^{(e)}(X)\right)\right]=\left[\pi_{c e}\left(\mathcal{L}^{(e)}(X)\right)\right] \mathbf{L}^{d(n-c e)}
$$


Proof. - Since (b) is a direct consequence of (a), we only have to prove (a). We may assume $X$ is affine, say $X=\operatorname{Spec}\left(k\left[x_{1}, \ldots, x_{N}\right] / I\right) \subset \mathbf{A}_{k}^{N}$. Clearly $X_{\text {sing }}$ is the intersection of hypersurfaces having an equation of the form $h \delta=0$ where $h$ belongs to $k\left[x_{1}, \ldots, x_{N}\right]$ and $\delta$ is some $N-d$ by $N-d$ minor of the matrix $\Delta:=\frac{\partial\left(f_{1}, \ldots, f_{N-d}\right)}{\partial\left(x_{1}, \ldots, x_{N}\right)}$, with $f_{1}, \ldots, f_{N-d}$ in $I$ and $h I \subset\left(f_{1}, \ldots, f_{N-d}\right)$. Hence, by Hilbert's Nullstellensatz, there exists $c$ in $\mathbf{N} \backslash\{0\}$ such that, for any $e$ in $\mathbf{N}$, the set $\mathcal{L}^{(e)}(X)$ is covered by a finite number of sets of the form

$$
A:=\left\{\varphi \in \mathcal{L}\left(\mathbf{A}_{k}^{N}\right) \mid(h \delta)(\tilde{\varphi}) \not \equiv 0 \bmod t^{c e+1}\right\} .
$$

Thus it is sufficient to prove that, for $n \geq c e$, the map $\theta_{n}$ is a piecewise trivial fibration over $\pi_{n}(\mathcal{L}(X) \cap A)$ with fiber $\mathbf{A}_{k}^{d}$. Since $h(\tilde{\varphi}) \neq 0$ for all $\varphi$ in $A$,

$$
\mathcal{L}(X) \cap A=\mathcal{L}\left(\operatorname{Spec} k\left[x_{1}, \ldots, x_{N}\right] /\left(f_{1}, \ldots, f_{N-d}\right)\right) \cap A .
$$

Hence we may assume $I=\left(f_{1}, \ldots f_{N-d}\right)$, i.e. $X$ is a complete intersection. Let $e^{\prime} \in \mathbf{N}, e^{\prime} \leq c e$, and set

$A^{\prime}:=\left\{\varphi \in A \mid \operatorname{ord}_{t} \delta(\tilde{\varphi})=e^{\prime}\right.$ and $\operatorname{ord}_{t} \delta^{\prime}(\tilde{\varphi}) \geq e^{\prime}$ for all $N-d$ by $N-d \operatorname{minors} \delta^{\prime}$ of $\left.\Delta\right\}$.

It is sufficient to prove that the map $\theta_{n}$ is a piecewise trivial fibration over $\pi_{n}(\mathcal{L}(X) \cap$ $\left.A^{\prime}\right)$ with fiber $\mathbf{A}_{k}^{d}$. We may assume $\delta$ is the minor of the first $N-d$ columns of $\Delta$. Let $s:\left(\bar{k}[t] / t^{n+1}\right)^{N} \rightarrow \bar{k}[t]^{N}$ be the $\bar{k}$-linear map given by $t^{\ell} \bmod t^{n+1} \mapsto t^{\ell}$ for $\ell=0,1, \ldots, n$. Let $\bar{\varphi} \in\left(\bar{k}[t] / t^{n+1}\right)^{N}$ be any $\bar{k}$-rational point of $\pi_{n}\left(\mathcal{L}(X) \cap A^{\prime}\right)$. We have

$$
\theta_{n}^{-1}(\bar{\varphi})=\left\{s(\bar{\varphi})+t^{n+1} y \bmod t^{n+2} \mid y \in \bar{k}[[t]]^{N}, f\left(s(\bar{\varphi})+t^{n+1} y\right)=0\right\},
$$

where $f$ is the column with entries $f_{1}, \ldots, f_{N-d}$. By Taylor expansion, the condition $f\left(s(\bar{\varphi})+t^{n+1} y\right)=0$ can be rewritten as

$$
f(s(\bar{\varphi}))+t^{n+1} \Delta(s(\bar{\varphi})) y+t^{2(n+1)}(\cdots)=0 .
$$

There exists an $N-d$ by $N-d$ matrix $M$ over $k\left[x_{1}, \ldots, x_{N}\right]$, independent of the choice of $\bar{\varphi}$, such that

$$
M \Delta=\left(\delta I_{N-d}, W\right),
$$

where $I_{N-d}$ is the identity matrix with $N-d$ columns and $W$ is an $N-d$ by $d$ matrix such that $W(s(\bar{\varphi})) \equiv 0 \bmod t^{e^{\prime}}$. Indeed, to check this last congruence, one expresses the last $d$ columns of $\Delta$ in terms of the first $N-d$ columns by Cramer's rule and then one uses the definition of $A^{\prime}$.

Condition (1) is equivalent to

$$
t^{-e^{\prime}-n-1}(M f)(s(\bar{\varphi}))+t^{-e^{\prime}}(M \Delta)(s(\bar{\varphi})) y+t^{n+1-e^{\prime}}(\cdots)=0 .
$$

Note that $t^{-e^{\prime}}(M \Delta)(s(\bar{\varphi}))$ is a matrix over $\bar{k}[[t]]$, whose minor determined by the first $N-d$ columns is not divisible by $t$, because $\operatorname{ord}_{t} \delta(\tilde{\varphi})=e^{\prime}$. Moreover, $n+1-e^{\prime} \geq 1$. Since $\bar{\varphi}$ is liftable to $\mathcal{L}(X)$ (i.e. belongs to $\pi_{n}\left(\mathcal{L}(X)\right.$ ), equation $\left(1^{\prime}\right)$ has a solution $y$ 
in $\bar{k}[[t]]^{N}$, and thus $t^{-e^{\prime}-n-1}(M f)(s(\bar{\varphi}))$ is a column matrix over $\bar{k}[[t]]$. By Hensel's Lemma, we deduce that $\theta_{n}^{-1}(\bar{\varphi})$ is equal to the set of all $s(\bar{\varphi})+t^{n+1} y_{0}$, with $y_{0}$ in $\bar{k}^{N}$ such that

$$
t^{-e^{\prime}-n-1}(M f)(s(\bar{\varphi}))+t^{-e^{\prime}}(M \Delta)(s(\bar{\varphi})) y_{0} \equiv 0 \bmod t .
$$

Thus the fiber $\theta_{n}^{-1}(\bar{\varphi})$ is a $d$-dimensional affine subspace of $\mathbf{A}_{k}^{N}$, given by linear equations which express the first $N-d$ coordinates in terms of linear combinations of the last $d$ coordinates, with coefficients which are regular functions on each locally closed subset of $\mathcal{L}_{n}(X)$ contained in $\pi_{n}\left(\mathcal{L}(X) \cap A^{\prime}\right)$. This proves that $\theta_{n}$ is a piecewise trivial fibration over $\pi_{n}\left(\mathcal{L}(X) \cap A^{\prime}\right)$ with fiber $\mathbf{A}_{k}^{d}$.

Assume now $X=\operatorname{Spec}\left(k\left[x_{1}, \ldots, x_{N}\right] /\left(f_{1}, \ldots, f_{N-d}\right)\right)$, with $f_{1}, \ldots, f_{N-d}$ in $k\left[x_{1}, \ldots, x_{N}\right]$. Fix $m, n, e$ in $\mathbf{N}$ with $m>n \geq e$. Set

$$
\Delta:=\frac{\partial\left(f_{1}, \ldots, f_{N-d}\right)}{\partial\left(x_{1}, \ldots, x_{N}\right)}, \quad \delta:=\operatorname{det} \frac{\partial\left(f_{1}, \ldots, f_{N-d}\right)}{\partial\left(x_{1}, \ldots, x_{N-d}\right)},
$$

and

$A:=\left\{\varphi \in \mathcal{L}(X) \mid \operatorname{ord}_{t} \delta(\tilde{\varphi})=e \leq \operatorname{ord}_{t} \delta^{\prime}(\tilde{\varphi})\right.$ for every $N-d$ by $N-d$ minor $\delta^{\prime}$ of $\left.\Delta\right\}$.

Let $p: X \rightarrow \mathbf{A}_{k}^{d}$ denote the projection onto the last $d$ coordinates. Denote by $\kappa$ the natural map $\kappa: \pi_{m}(A) \rightarrow \pi_{n}(A) \times_{\mathcal{L}_{n}\left(\mathbf{A}_{k}^{d}\right)} \mathcal{L}_{m}\left(\mathbf{A}_{k}^{d}\right)$ induced by $\varphi \in \mathcal{L}(X) \mapsto$ $\left(\pi_{n}(\varphi), p(\varphi)\right)$, where the fiber product is with respect to the map $\left.\pi_{n}(A) \subset \mathcal{L}_{n}(X)\right) \rightarrow$ $\mathcal{L}_{n}\left(\mathbf{A}_{k}^{d}\right)$ induced by $p$.

Lemma 4.2. - The map $\kappa$ is a bijection. Moreover, for any locally closed subvariety $Z$ of $\mathcal{L}_{n}(X) \times_{\mathcal{L}_{n}\left(\mathbf{A}_{k}^{d}\right)} \mathcal{L}_{m}\left(\mathbf{A}_{k}^{d}\right)$ contained in the domain of $\kappa^{-1}$, the restriction of $\kappa^{-1}$ to $Z$ is a morphism from $Z$ to $\mathcal{L}_{m}(X)$.

Proof. - By induction it is enough to treat the case where $m=n+1$, but this case is just a reformulation of material in the proof of Lemma 4.1.

Lemma 4.3. - Let $X$ be an algebraic variety over $k$ of dimension $d$.

(1) For any $n$ in $\mathbf{N}$,

$$
\operatorname{dim} \pi_{n}(\mathcal{L}(X)) \leq(n+1) d .
$$

(2) For any $n, m$ in $\mathbf{N}$, with $m \geq n$, the fibers of $\pi_{m}(\mathcal{L}(X)) \rightarrow \pi_{n}(\mathcal{L}(X))$ are of dimension $\leq(m-n) d$.

Proof. - This lemma is probably well known and is implicit in [O]. Assertion (1) follows from assertion (2). Moreover, it suffices to prove (2) for $m=n+1$, and we may assume that $X$ is affine and that $k=\bar{k}$. Substituting $a_{i}+t^{n+1} x_{i}$ for the affine coordinates $x_{i}$ in the equations defining $X$ in some affine space, we see that each fiber of $\pi_{n+1}(\mathcal{L}(X)) \rightarrow \pi_{n}(\mathcal{L}(X))$ is contained in the reduction mod $t$ of a scheme which is flat and of finite type over $\operatorname{Spec}(k[t])$ with generic fiber $X \otimes_{k} k(t)$. But the reduction mod $t$ of such a scheme has dimension at most $d$. 
(4.4) Let $X$ be an algebraic variety over $k$. By Greenberg's theorem [G], for any $n$ in $\mathbf{N}$ there exists $\gamma(n) \geq n$ in $\mathbf{N}$ such that $\pi_{n}(\mathcal{L}(X))$ is the image of $\mathcal{L}_{\gamma(n)}(X)$ by the natural projection and which is minimal for this property. Furthermore the function $\gamma(n)$ is bounded by a real linear function of $n$. We call $\gamma$ the Greenberg function for $X$. It has been studied in $[\mathrm{L}-\mathrm{J}],[\mathrm{H}]$.

LEMMA 4.4. - Let $X$ be an algebraic variety over $k$ of dimension d and let $S$ be a closed subvariety of dimension $<d$. Let $\gamma$ be the Greenberg function for $S$. For any $n$, $i$, e in $\mathbf{N}$, with $n \geq i \geq \gamma(e), \pi_{n, X}\left(\pi_{i, X}^{-1} \mathcal{L}_{i}(S)\right)$ is of dimension $\leq(n+1) d-e-1$.

Proof. - We drop the subscript $X$ in $\pi_{n}$, etc. Clearly we may assume $i=\gamma(e)$. By Lemma 4.3 (2) applied to the projection

$$
\pi_{n}\left(\pi_{\gamma(e)}^{-1} \mathcal{L}_{\gamma(e)}(S)\right) \rightarrow \pi_{e}\left(\pi_{\gamma(e)}^{-1} \mathcal{L}_{\gamma(e)}(S)\right)
$$

we obtain

$$
\operatorname{dim} \pi_{n}\left(\pi_{\gamma(e)}^{-1} \mathcal{L}_{\gamma(e)}(S)\right) \leq(n-e) d+\operatorname{dim} \pi_{e}\left(\pi_{\gamma(e)}^{-1} \mathcal{L}_{\gamma(e)}(S)\right) .
$$

Since, by definition of the Greenberg function, $\pi_{e}\left(\pi_{\gamma(e)}^{-1} \mathcal{L}_{\gamma(e)}(S)\right)=\pi_{e}(\mathcal{L}(S))$, the result follows because, by Lemma $4.3(1), \operatorname{dim} \pi_{e}(\mathcal{L}(S)) \leq(e+1)(d-1)$.

\section{Proof of Theorem 1.1 and rationality results}

THEOREM 5.1. - Let $X$ be an algebraic variety over $k$ of pure dimension $d$. Let $A_{n}, n \in \mathbf{Z}^{r}$, be a semi-algebraic family of semi-algebraic subsets of $\mathcal{L}(X)$ and let $\alpha: \mathcal{L}(X) \times \mathbf{Z}^{r} \rightarrow \mathbf{N}$ be a simple function. Then the power series

$$
\sum_{n \in \mathbf{N}^{r}} T^{n} \int_{A_{n}} \mathbf{L}^{-\alpha(-, n)} d \mu
$$

in the variable $T=\left(T_{1}, \ldots, T_{r}\right)$ belongs to the subring of $\widehat{\mathcal{M}}[[T]]$ generated by the image in $\widehat{\mathcal{M}}[[T]]$ of $\mathcal{M}_{\text {loc }}[T],\left(\mathbf{L}^{i}-1\right)^{-1}$ and $\left(1-\mathbf{L}^{-a} T^{b}\right)^{-1}$, with $i \in \mathbf{N} \backslash\{0\}, a \in \mathbf{N}$, $b \in \mathbf{N}^{r} \backslash\{0\}$.

Corollary. - For any semi-algebraic subset $A$ of $\mathcal{L}(X)$, the measure $\mu(A)$ is in $\overline{\mathcal{M}}_{\text {loc }}\left[\left(\left(\mathbf{L}^{i}-1\right)^{-1}\right)_{i \geq 1}\right]$, where $\overline{\mathcal{M}}_{\text {loc }}$ is the image of $\mathcal{M}_{\text {loc }}$ in $\widehat{\mathcal{M}}$, cf. (3.2).

THEOREM 5.1'. - Let $X$ be an algebraic variety over $k$ of pure dimension $d$. Let $A_{n}, n \in \mathbf{Z}^{r}$, be a semi-algebraic family of semi-algebraic subsets of $\mathcal{L}(X)$ and let $\alpha: \mathcal{L}(X) \times \mathbf{Z}^{r} \rightarrow \mathbf{N}$ be a simple function. Assume that $A_{n} \cap \mathcal{L}\left(X_{\text {sing }}\right)=\emptyset$ and that $A_{n}$ and the fibers of $\alpha\left({ }_{-}, n\right): A_{n} \rightarrow \mathbf{N}$ are weakly stable (and hence stable), for every $n \in \mathbf{N}^{r}$. Then the power series

$$
\sum_{n \in \mathbf{N}^{r}} T^{n} \int_{A_{n}} \mathbf{L}^{-\alpha(-, n)} d \tilde{\mu}
$$

in the variable $T=\left(T_{1}, \ldots, T_{r}\right)$ belongs to the subring of $\mathcal{M}_{\mathrm{loc}}[[T]]$ generated by $\mathcal{M}_{\text {loc }}[T]$ and the series $\left(1-\mathbf{L}^{-a} T^{b}\right)^{-1}$, with $a \in \mathbf{N}$ and $b \in \mathbf{N}^{r} \backslash\{0\}$. 
Proof of Theorem 5.1'. — Using a resolution of singularities $\pi: \tilde{X} \rightarrow X$, with exceptional locus $\pi^{-1}\left(X_{\text {sing }}\right)$ and using Lemma 3.3, we may assume that $X$ is smooth. Moreover we may also assume that $X$ is affine. For $n$ in $\mathbf{N}^{r}, m$ in $\mathbf{N}$, set

$$
A_{n, m}:=\left\{x \in A_{n} \mid \alpha(x, n)=m\right\} .
$$

For every $n$, the map $\alpha\left({ }_{-}, n\right): A_{n} \rightarrow \mathbf{N}$ is bounded, cf. (3.1). Hence by Theorem 2.1 there exists a Presburger function $\theta: \mathbf{Z}^{r} \rightarrow \mathbf{N}$ such that $\alpha(x, n) \leq \theta(n)$ for every $x \in A_{n}, n \in \mathbf{N}^{r}$. Thus the series (1) is equal to

$$
\sum_{\substack{n \in \mathbb{N}^{r}, m \in \mathbf{N} \\ m \leq \theta(n)}} \tilde{\mu}\left(A_{n, m}\right) \mathbf{L}^{-m} T^{n} .
$$

By Lemma 2.8, the family $\left(A_{n, m}\right)_{n \in \mathbf{N}^{r}, m \in \mathbf{N}}$ is a finite boolean combination of semialgebraic families which have bounded representations. Recall that each member of a family with bounded representation is weakly stable and hence stable, because $X$ is smooth. Using that $\tilde{\mu}\left(A^{\prime} \cup A^{\prime \prime}\right)=\tilde{\mu}\left(A^{\prime}\right)+\tilde{\mu}\left(A^{\prime \prime}\right)-\tilde{\mu}\left(A^{\prime} \cap A^{\prime \prime}\right)$, we may assume that the family $\left(A_{n, m}\right)_{n, m}$ is a finite intersection of families each of which has the property that it or its complement has bounded representation. Using that $\tilde{\mu}\left(A^{\prime} \backslash A^{\prime \prime}\right)=$ $\tilde{\mu}\left(A^{\prime}\right)-\tilde{\mu}\left(A^{\prime} \cap A^{\prime \prime}\right)$, we may assume that the family $\left(A_{n, m}\right)_{n, m}$ is a finite union of finite intersections of families with bounded representation. Since a finite intersection of families with bounded representation has bounded representation, we may finally assume that the family $\left(A_{n, m}\right)_{n, m}$ has bounded representation. We may further assume that in the bounded representation (2.8) of $\left(A_{n, m}\right)_{n, m}$, no covering by affine open subsets $U$ is needed. Let $F$ be the product of all the functions $f_{i}$ (assumed to be regular on $X$ and to have bounded order on each $A_{n, m}$ ) appearing in the conditions of the form 2.1 (i), (ii) and (iii) in the bounded representation of the family $\left(A_{n, m}\right)_{n, m}$.

Let $h: Y \rightarrow X$ be an embedded resolution of singularities of the locus of $F=0$ in $X$. The exceptional locus of $h$ is contained in $h^{-1}\left(F^{-1}(0)\right)$. We can cover $Y$ by affine open subsets $U$ on which there exist regular functions $z_{1}, \ldots, z_{d}$ inducing an étale map $U \rightarrow \mathbf{A}_{k}^{d}$ such that on $U$ each $f_{i} \circ h$ is a monomial in $z_{1}, \ldots, z_{d}$ multiplied by a regular function with no zeros on $U$. For such an open subset $U$ we may assume that the $z_{i}$ 's appearing in at least one of these monomials are exactly $z_{1}, z_{2}, \ldots$, $z_{d_{0}}$. Since $\operatorname{ord}_{t} F$ is bounded on $A_{n, m}$, Lemma 3.3 yields that, uniformly in $n, m$, $\tilde{\mu}\left(A_{n, m}\right)$ is a finite $\mathbf{Z}$-linear combination of terms of the form

$$
\sum_{\substack{\ell_{1}, \ldots, \ell_{d_{0}} \in \mathbf{N} \\ \theta\left(\ell_{1}, \ldots, \ell_{d_{0}}, n, m\right)}} \mathbf{L}^{-\beta\left(\ell_{1}, \ldots, \ell_{d_{0}}\right)} \tilde{\mu}\left(W_{\ell_{1}, \ldots, \ell_{d_{0}}}\right),
$$

where $\theta\left(\ell_{1}, \ldots, \ell_{d_{0}}, n, m\right)$ is a semi-algebraic condition defining a Presburger subset of $\mathbf{Z}^{d_{0}+r+1}, \beta$ is a linear form with coefficients in $\mathbf{N}$, and $W_{\ell_{1}, \ldots, \ell_{d_{0}}}$ is of the form

$$
\left\{y \in \mathcal{L}(U) \mid \operatorname{ord}_{t} z_{i}=\ell_{i} \text { for } i=1, \ldots, d_{0}, \quad \text { and } \quad\left(\overline{a c}\left(z_{1}\right), \ldots, \overline{a c}\left(z_{d_{0}}\right), \pi_{0}(y)\right) \in W\right\},
$$

with $W$ a constructible subset of $\left(\mathbf{A}_{k}^{1} \backslash\{0\}\right)^{d_{0}} \times U$ and $U$ as above. (Recall that $\pi_{0}: \mathcal{L}(Y) \rightarrow \mathcal{L}_{0}(Y)=Y$ is the natural projection.) Note that the sum in (3) is finite for each fixed $n, m$, since $\operatorname{ord}_{t} F$ is bounded on $A_{n, m}$. 
From Lemma 4.2 with $n=e=0$ we obtain

$$
\tilde{\mu}\left(W_{\ell_{1}, \ldots, \ell_{d_{0}}}\right)=\left[W^{\prime}\right] \mathbf{L}^{-\left(\sum_{i=1}^{d_{0}} \ell_{i}\right)-d}
$$

where $W^{\prime}$ is the set of $\left(w_{1}, \ldots, w_{d_{0}}, y\right)$ 's in $W \subset\left(\mathbf{A}_{k}^{1} \backslash\{0\}\right)^{d_{0}} \times U$ such that $z_{i}(y)=0$ when $\ell_{i}>0$ and $z_{i}(y)=w_{i}$ when $\ell_{i}=0$. We conclude by (2), (3) and (4), that the series (1) is a finite $\mathcal{M}_{\text {loc }}$-linear combination of series $f\left(\mathbf{L}^{-1}, T_{1}, \ldots, T_{r}\right)$, with $f\left(X_{1}, X_{2}, \ldots, X_{r+1}\right)$ in $\mathbf{Z}\left[X_{1}\right]\left[\left[X_{2}, \cdots, X_{r+1}\right]\right]$ given as in Lemma 5.2 below (with $r$ replaced by $r+1$ ). The theorem follows now directly from Lemma 5.2 and Lemma 5.3 below.

Proof of Theorem 5.1. - The argument is the same as in the proof of Theorem 5.1', but easier. Now we do not have to work with bounded representations anymore, since $\widehat{\mathcal{M}}$ is complete, and we are allowed to work with infinite sums (which have no meaning in $\mathcal{M}_{\text {loc }}$ ). Finally we obtain that the series (1) is a finite $\overline{\mathcal{M}}_{\text {loc }}$-linear combination of series $f\left(\mathbf{L}^{-1}, T_{1}, \ldots, T_{r}\right)$, with $f\left(X_{1}, X_{2}, \ldots, X_{r+1}\right)$ in $\mathbf{Z}\left[\left[X_{1}, \cdots, X_{r+1}\right]\right]$ given as in Lemma 5.2 below (with $r$ replaced by $r+1)$. However the series $f\left(X_{1}, X_{2}, \ldots, X_{r+1}\right)$ might not be in $\mathbf{Z}\left[X_{1}\right]\left[\left[X_{2}, \cdots, X_{r+1}\right]\right]$, so we cannot apply Lemma 5.3 (as in the proof of Theorem $5.1^{\prime}$ ). We leave the details to the reader.

Lemma 5.2. - Let $P$ be a Presburger subset of $\mathbf{Z}^{m}$ and let $\varphi_{1}: \mathbf{Z}^{m} \rightarrow \mathbf{N}, \ldots$, $\varphi_{r}: \mathbf{Z}^{m} \rightarrow \mathbf{N}$ be Presburger functions. Assume that the fibers of the map $\varphi: P \rightarrow \mathbf{N}^{r}$ given by $i \mapsto\left(\varphi_{1}(i), \ldots, \varphi_{r}(i)\right)$ are finite. Then the power series $f(X):=\sum_{i \in P} X^{\varphi(i)}$, in the variable $X=\left(X_{1}, \ldots, X_{r}\right)$, belongs to the subring of $\mathbf{Z}[[X]]$ generated by $\mathbf{Z}[X]$ and the series $\left(1-X^{c}\right)^{-1}$, with $c \in \mathbf{N}^{r} \backslash\{0\}$.

Proof. - We may assume that $P \subset \mathbf{N}^{m}$. We first consider the special case where $r=m$ and $\varphi(i)=i$. By replacing $i$ by $d i+a$ for suitable $d \in \mathbf{N} \backslash\{0\}, a \in \mathbf{N}^{m}$, we may assume that no congruence relations appear in the description of $P$. Since $\sum_{P_{1} \cup P_{2}}=\sum_{P_{1}}+\sum_{P_{2}}-\sum_{P_{1} \cap P_{2}}$, we may further assume that $P$ is the set of integral points in a rational convex polyhedron $\bar{P} \subset \mathbf{R}_{+}^{m}$. (Here $\mathbf{R}_{+}:=\{x \in \mathbf{R} \mid x \geq 0\}$.) A straightforward calculation yields the lemma when $\bar{P}$ is a polyhedral cone generated by part of a basis of $\mathbf{Z}^{n}$. Hence the lemma also holds in our special case when $\bar{P}$ is any rational convex polyhedral cone, since any such cone can be decomposed in cones generated by part of a basis of $\mathbf{Z}^{n}$ (see, e.g., [Da] p.123-124). Let $\bar{P}^{\prime} \subset \mathbf{R}_{+}^{m+1}$ be a rational convex polyhedral cone such that $\bar{P}$ is equal to the intersection of $\bar{P}^{\prime}$ with the hyperplane given by the equation $x_{m+1}=1$ in $\mathbf{R}^{m+1}$. Set

$$
g(X, T):=\sum_{\substack{i \in \mathbf{N}^{m}, \ell \in \mathbf{N} \\(i, \ell) \in \bar{P}^{\prime}}} X^{i} T^{\ell},
$$

where $T$ is a new variable. Since $\bar{P}^{\prime}$ is a polyhedral cone, we already know that $g(X, T)$ belongs to the subring of $\mathbf{Z}[[X, T]]$ generated by $\mathbf{Z}[X, T]$ and the series $\left(1-X^{c} T^{a}\right)^{-1}$, with $(c, a) \in \mathbf{N}^{r+1} \backslash\{0\}$. Since $f=\frac{\partial g}{\partial T}(X, 0)$, this proves Lemma 5.2 in the special case where $r=m$ and $\varphi(i)=i$. For the general case, consider

$$
h(Y, X):=\sum_{i \in P, j=\varphi(i)} Y^{i} X^{j}
$$


where $Y=\left(Y_{1}, \ldots, Y_{m}\right)$. The special case implies that $h(Y, X)$ belongs to the subring of $\mathbf{Z}[[Y, X]]$ generated by $\mathbf{Z}[Y, X]$ and the series $\left(1-Y^{b} X^{c}\right)^{-1}$ with $(b, c) \in \mathbf{N}^{m+r} \backslash\{0\}$. Note that $h(Y, X)$ belongs to $\mathbf{Z}[Y][[X]]$, since the fibers of $\varphi$ are finite. By Lemma 5.3 we can take in the above $c \neq 0$, which finishes the proof of Lemma 5.2, since $f(X)=h(1, X)$.

LEMmA 5.3. - Let $R$ be a commutative ring with unit. Let $Y=\left(Y_{1}, \ldots, Y_{m}\right)$ and $X=\left(X_{1}, \ldots, X_{r}\right)$ be variables. Assume that $h(Y, X)$ belongs to the subring of $R[[Y, X]]$ generated by $R[Y, X]$ and the series $\left(1-Y^{a} X^{b}\right)^{-1}$ with $(a, b) \in \mathbf{N}^{m+r} \backslash\{0\}$. If, moreover, $h(Y, X)$ belongs to $R[Y][[X]]$, then we can take in the above $b \neq 0$.

Proof. - Straightforward.

(5.4) Proof of Theorem 1.1.- We will prove the following theorem of which Theorem 1.1 is a special case.

THEOREM 5.4. - Let $X$ be an algebraic variety over $k$ and let $A$ be a semialgebraic subset of $\mathcal{L}(X)$. The power series

$$
P_{A}(T):=\sum_{n=0}^{\infty}\left[\pi_{n}(A)\right] T^{n},
$$

considered as an element of $\mathcal{M}_{\text {loc }}[[T]]$, is rational and belongs to $\mathcal{M}[T]_{\text {loc }}$.

Proof. - We may assume $X$ is a closed subvariety of a smooth connected algebraic variety $W$ of dimension $d$. That $P_{A}(T)$ is in $\mathcal{M}[T]_{\text {loc }}$ follows directly from Theorem $5.1^{\prime}$ because

$$
\left[\pi_{n, X}(A)\right] \mathbf{L}^{-(n+1) d}=\int_{\pi_{n, W}^{-1}\left(\pi_{n, X}(A)\right)} \mathbf{L}^{0} d \tilde{\mu}_{\mathcal{L}(W)}
$$

and the family $\pi_{n, W}^{-1}\left(\pi_{n, X}(A)\right), n \in \mathbf{N}$, is a semi-algebraic family of stable semi-algebraic subsets of $\mathcal{L}(W)$ by Theorem 2.1 .

Remark. - Note that the above proof of Theorem 5.4 only uses the material in $\S 3$ with $X$ smooth. Moreover it does not use (3.2).

\section{Formula for $\mu(\mathcal{L}(X))$ and applications}

(6.1) Let $S$ be an algebraic variety over C. Consider the Hodge numbers

$$
e^{p, q}(S):=\sum_{i \geq 0}(-1)^{i} h^{p, q}\left(H_{c}^{i}(S, \mathbf{C})\right)
$$

where $h^{p, q}\left(H_{c}^{i}(S, \mathbf{C})\right)$ denotes the rank of the $(p, q)$-Hodge component of the $i$-th cohomology group with compact supports. Define the Hodge polynomial of $S$ as

$$
H(S ; u, v):=\sum_{p, q} e^{p, q}(S) u^{p} v^{q}
$$


Note that $H\left(\mathbf{A}_{\mathbf{C}}^{1} ; u, v\right)=u v$ and that $\operatorname{deg} H(S ; u, v)=2 \operatorname{dim} S$. The map $S \mapsto$ $H(S ; u, v)$ factors through $\mathcal{M}$ and induces a ring morphism $H: \mathcal{M} \rightarrow \mathbf{Z}[u, v]$, which extends naturally to a ring morphism $H: \mathcal{M}_{\text {loc }} \rightarrow \mathbf{Z}[u, v]\left[(u v)^{-1}\right]$. Clearly $\operatorname{deg} H(Z ; u, v) \leq-2 m$ when $Z \in F^{m} \mathcal{M}_{\text {loc }}$, hence the kernel of the natural map $\mathcal{M}_{\text {loc }} \rightarrow \widehat{\mathcal{M}}$ is killed by $H$, and $H$ induces a ring morphism

$$
H: \overline{\mathcal{M}}_{\mathrm{loc}} \longrightarrow \mathbf{Z}[u, v]\left[(u v)^{-1}\right],
$$

where $\overline{\mathcal{M}}_{\text {loc }}$ is the image of $\mathcal{M}_{\text {loc }}$ in $\widehat{\mathcal{M}}$.

We denote by $\chi(S)$ the topological Euler characteristic of $S$, i.e. the alternating sum of the rank of its Betti or de Rham cohomology groups. Clearly $\chi(S)=$ $H(S ; 1,1)$. Hence the map $S \mapsto \chi(S)$ factors through $\overline{\mathcal{M}}_{\text {loc }}$ and induces a ring morphism $\chi: \overline{\mathcal{M}}_{\text {loc }} \rightarrow \mathbf{Z}$. Such morphisms $H$ and $\chi$ can also be defined when $k \neq \mathbf{C}$, using an embedding into $\mathbf{C}$ of the field of definition of $S$. Indeed, the $e^{p, q}(S)$ 's do not depend on the embedding, since for a smooth projective variety $S$ they are equal to $(-1)^{p+q} \operatorname{dim} H^{q}\left(S, \Omega_{S}^{p}\right)$.

More generally let $\mathrm{Mot}_{k}$ denote the category of Chow motives over $k$. It follows from $[\mathrm{G}-\mathrm{S}]$ and $[\mathrm{G}-\mathrm{N}]$ that there exists a natural morphism $\mathcal{M}_{\mathrm{loc}} \rightarrow K_{0}\left(\mathrm{Mot}_{k}\right)$. The conjectural existence of a weight filtration (with the expected properties) on the objects of the category Mot $_{k}$ would imply that this morphism factors through $\overline{\mathcal{M}}_{\text {loc }}$. In particular, without using any conjecture, this is true when we replace Chow motives by their étale or Hodge realizations.

(6.2) Let $X$ be an algebraic variety over $k$ of pure dimension $d$, and let $h: Y \rightarrow X$ be a resolution of $X$. By this we mean $Y$ is a smooth algebraic variety over $k, h$ is birational, proper and defined over $k$, and the exceptional locus $E$ of $h$ has normal crossings, meaning that the $k$-irreducible components of $E$ are smooth and intersect transversally. Let us denote the $k$-irreducible components of $E$ by $E_{i}, i \in J$. For $I \subset J$, set $E_{I}=\bigcap_{i \in I} E_{i}$ and $E_{I}^{\circ}=E_{I} \backslash \bigcup_{j \notin I} E_{j}$. Note that the support of the subscheme defined by the ideal sheaf $\mathcal{I}\left(h^{*}\left(\Omega_{X}^{d}\right)\right)$ is contained in $E$. (See (3.3) for the notation $\mathcal{I}\left({ }_{-}\right)$.)

(6.3) Assume the hypothesises of (6.2) hold and moreover that the ideal sheaf $\mathcal{I}\left(h^{*}\left(\Omega_{X}^{d}\right)\right)$ is invertible. Such a resolution $h$ always exists by Hironaka's Theorem. For $i$ in $I$, set

(6.3.1) $\nu_{i}=1+\left(\right.$ multiplicity of $E_{i}$ in the divisor of $Y$ determined by $\left.\mathcal{I}\left(h^{*}\left(\Omega_{X}^{d}\right)\right)\right)$.

Proposition 6.3.2. - Let $W$ be a closed subvariety of $X$. The following formula holds in $\widehat{\mathcal{M}}$,

$$
\mu_{\mathcal{L}(X)}\left(\pi_{0}^{-1}(W)\right)=\mathbf{L}^{-d} \sum_{I \subset J}\left[E_{I}^{\circ} \cap h^{-1}(W)\right] \prod_{i \in I} \frac{\mathbf{L}-1}{\mathbf{L}^{\nu_{i}}-1}
$$


Proof. — It follows directly from Lemma 3.3 (cf. the proof of 5.1).

Corollary 6.3.3. - Let $X$ be a variety over $k$ of pure dimension $d$. Then $\mu(\mathcal{L}(X))$ belongs to $\overline{\mathcal{M}}_{\text {loc }}\left[\left(\frac{\mathbf{L}-1}{\mathbf{L}^{i}-1}\right)_{i \geq 1}\right]$.

The morphism $\chi: \overline{\mathcal{M}}_{\text {loc }} \rightarrow \mathbf{Z}$ defined in (6.1) may be extended uniquely to a ring morphism $\chi: \overline{\mathcal{M}}_{\text {loc }}\left[\left(\frac{\mathbf{L}-1}{\mathbf{L}^{i}-1}\right)_{i \geq 1}\right] \rightarrow \mathbf{Q}$ by requiring $\chi\left(\frac{\mathbf{L}-1}{\mathbf{L}^{i}-1}\right)=\frac{1}{i}$ for $i \geq 1$.

Corollary 6.3.4. - Let $X$ be a variety over $k$ of pure dimension $d$. Then the Euler characteristic $\chi(\mu(\mathcal{L}(X)))$ is well defined as a rational number.

Similarly, the morphism $H: \overline{\mathcal{M}}_{\text {loc }} \rightarrow \mathbf{Z}[u, v]\left[(u v)^{-1}\right]$ defined in (6.1) may be extended naturally to a morphism of rings

$$
H: \overline{\mathcal{M}}_{\mathrm{loc}}\left[\left(\frac{\mathbf{L}-1}{\mathbf{L}^{i}-1}\right)_{i \geq 1}\right] \longrightarrow \mathbf{Z}[u, v]\left[(u v)^{-1}\right]\left[\left(\frac{u v-1}{(u v)^{i}-1}\right)_{i \geq 1}\right] .
$$

So if $X$ is a variety over $\mathbf{C}$ of pure dimension $d$, we have $H(\mu(\mathcal{L}(X)) ; u, v)$ which is well defined in $\mathbf{Z}[u, v]\left[(u v)^{-1}\right]\left[\left(\frac{u v-1}{(u v)^{i}-1}\right)_{i \geq 1}\right]$.

(6.4) Assume the hypothesises of (6.2) hold. Instead of requiring that $\mathcal{I}\left(h^{*}\left(\Omega_{X}^{d}\right)\right)$ is invertible we will now assume $\mathcal{I}\left(h^{*}\left(\Omega_{X}^{d}\right)\right)$ is locally generated by elements whose zero loci are contained in $E$. This weaker condition is more practical for explicit computations. To $x$ in $E_{I}^{\circ}, I \subset J$, we associate a polyhedron $\Delta_{x} \subset \mathbf{R}^{|I|}$ as follows. For $i$ in $I$ and $g$ a section of $\mathcal{I}\left(h^{*}\left(\Omega_{X}^{d}\right)\right)$ on an open set of $Y$ intersecting $E_{i}$, set

$$
\nu_{i}(g)=1+\left(\text { multiplicity of } E_{i} \text { in the divisor of } g\right) .
$$

Set

$$
\Delta_{x}:=\text { convex hull of } \bigcup_{g}\left(\left(\nu_{i}(g)\right)_{i \in I}+\mathbf{R}_{+}^{|I|}\right),
$$

where $g$ runs over all local sections at $x$ of $\mathcal{I}\left(h^{*}\left(\Omega_{X}^{d}\right)\right)$ whose zero loci are contained in $E$, and $\mathbf{R}_{+}=\{a \in \mathbf{R} \mid a \geq 0\}$. On each $k$-irreducible component $C$ of $E_{I}^{\circ}, \Delta_{x}$ is constant with value say $\Delta_{C}$. The support function $\ell(-, \Delta)$ of a polyhedron $\Delta$ in the interior of $\mathbf{R}_{+}^{|I|}$ is given, for $\xi$ in $\mathbf{R}_{+}^{|I|}$, by

$$
\ell(\xi, \Delta):=\min _{v \in \Delta} \xi \cdot v \text {. }
$$

We set

$$
Z(\Delta):=(\mathbf{L}-1)^{|I|} \sum_{\xi \in\left(\mathbf{N}^{\times}\right)^{|I|}} \mathbf{L}^{-\ell(\xi, \Delta)} \in \widehat{\mathcal{M}}
$$

where $\mathbf{N}^{\times}=\mathbf{N} \backslash\{0\}$. Note that $\left(\mathbf{N}^{\times}\right)^{|I|}$ may be partitioned into finitely many sets $C$ of the form $\mathbf{N}^{\times} \eta_{C, 1}+\cdots+\mathbf{N}^{\times} \eta_{C, e_{C}}$, with $\eta_{C, 1}, \ldots, \eta_{C, e_{C}}$ in $\mathbf{N}^{|I|}$ linearly independent, on which $\ell(-, \Delta)$ is linear. Thus

$$
Z(\Delta)=(\mathbf{L}-1)^{|I|} \sum_{C} \prod_{i=1, \ldots, e_{C}}\left(\mathbf{L}^{\ell\left(\eta_{C, i}, \Delta\right)}-1\right)^{-1} .
$$


Proposition 6.4.6. — Let $W$ be a closed subvariety of $X$. The following formula holds in $\widehat{\mathcal{M}}$,

$$
\mu_{\mathcal{L}(X)}\left(\pi_{0}^{-1}(W)\right)=\mathbf{L}^{-d} \sum_{C}\left[C \cap h^{-1}(W)\right] Z\left(\Delta_{C}\right)
$$

where $C$ runs over all $k$-irreducible components of $E_{I}^{\circ}$, for all $I \subset J$.

Proof. - It follows directly from (6.4.4) and Lemma 3.3 (cf. the proof of 5.1).

(6.5) Let $\mathcal{I}$ be an invertible ideal sheaf on $X$ and let $h: Y \rightarrow X$ be a resolution of $X$ such that both $E$ and the locus of $\mathcal{I O}_{Y}$ are contained in a divisor with normal crossings $E^{\prime}$ in $Y$ (cf. the notation in (6.2)). The previous results may be extended directly to integrals of the form $\int_{\pi_{0}^{-1}(W)} \mathbf{L}^{-\operatorname{ord}_{t} \mathcal{I}} d \mu$. More precisely, change notation from (6.2) so that the $E_{i}$ 's, $i \in J$, denote now the $k$-irreducible components of $E^{\prime}$. Let $N_{i}$ be the multiplicity of $E_{i}$ in the divisor of $\mathcal{I} \mathcal{O}_{Y}$. Replace $\nu_{i}$ in (6.3), resp. $\nu_{i}(g)$ in (6.4), by $\nu_{i}+N_{i}$, resp. $\nu_{i}(g)+N_{i}$. Then $\int_{\pi_{0}^{-1}(W)} \mathbf{L}^{-\operatorname{ord}_{t} \mathcal{I}} d \mu$ equals the right hand side of (6.3.2), resp. (6.4.6), when the hypothesis of (6.3), resp. (6.4), with $E$ replaced by $E^{\prime}$, is satisfied.

(6.6) Remark. - Assume $X$ is normal and let $\omega_{X}$ be the canonical sheaf of $X$. By definition $\omega_{X}:=j_{*}\left(\Omega_{X^{\circ}}^{d}\right)$, where $j: X^{\circ} \hookrightarrow X$ denotes the inclusion of the smooth locus of $X$. Assume moreover $X$ is Gorenstein, i.e. $\omega_{X}$ is invertible, and that all singularities of $X$ are canonical, meaning that there exists a resolution $h: Y \rightarrow X$ with $h^{*}\left(\omega_{X}\right) \subset \Omega_{Y}^{d}$. In this case Kontsevich $[\mathrm{K}]$ introduced the invariant

$$
\int_{\mathcal{L}(Y)} \mathbf{L}^{-\operatorname{ord}_{t} h^{*}\left(\omega_{X}\right)} d \mu_{Y}
$$

which does not depend on the resolution $h$. Indeed the independence follows from Lemma 3.3 (with both varieties smooth) and the fact that two resolutions are always dominated by a third one. If the resolution is crepant $\left(\right.$ i.e. $\left.h^{*}\left(\omega_{X}\right)=\Omega_{Y}^{d}\right)$, then (6.6.1) equals $\mathbf{L}^{-d} \sum_{I \subset J}\left[E_{I}^{\circ}\right]=\mathbf{L}^{-d}[Y]$ because of (6.3.2). In this way Kontsevich $[\mathrm{K}]$ showed that $H(Y ; u, v)$ is independent of the crepant resolution $h$ (assuming such a resolution exists). This independence was conjectured in [B-D]. For some related constructions, see $[\mathrm{V}]$.

\section{Motivic volume as a limit}

The following result is an analogue of a result by Oesterlé [O].

TheOREM 7.1. - Let $X$ be an algebraic variety over $k$ of pure dimension $d$. Let $A$ be a semi-algebraic subset of $\mathcal{L}(X)$. The sequence $\left[\pi_{n}(A)\right] \mathbf{L}^{-(n+1) d}$ converges in $\widehat{\mathcal{M}}$ to $\mu(A)$. Furthermore when $A=\mathcal{L}(X)$ this limit is nonzero. 
Proof. - By 4.4 and Lemma 4.3 we may assume that $X$ is affine and irreducible and that $A$ is given by a semi-algebraic condition. As in the proof of Lemma 3.1, let $g$ be a nonzero regular function on $X$ which vanishes on the singular locus of $X$, let $F$ be the product of $g$ and all the functions $f_{i}$ (assumed to be regular and not identically zero on $X$ ) appearing in the conditions of the form 2.1 (i), (ii) and (iii) in the description of the semi-algebraic set $A$, and let $S$ be the locus of $F=0$ in $X$. For $e \geq 0$, set $A^{(e)}:=A \backslash \pi_{e, X}^{-1} \mathcal{L}_{e}(S)$. Let $\gamma$ be the Greenberg function for $S$. It follows from Lemma 4.4 that, for $n \geq \gamma(e)$,

$$
\left[\pi_{n}(A)\right] \mathbf{L}^{-(n+1) d}-\left[\pi_{n}\left(A^{(\gamma(e))}\right)\right] \mathbf{L}^{-(n+1) d}
$$

belongs to $F^{e+1} \mathcal{M}_{\text {loc }}$. On $A^{(\gamma(e))}$, the function $\operatorname{ord}_{t} F(x)$ is bounded by a multiple of $\gamma(e)$. Hence, since $X_{\text {sing }} \subset S$ and $A^{(e)} \subset \mathcal{L}^{(e)}(X)$, it follows from Lemma 4.1 that $A^{(\gamma(e))}$ is stable at level $c \gamma(e)$, for some $c \geq 1$ independent of $e$. Thus, for $n$ and $n^{\prime} \geq c \gamma(e)$,

$$
\left[\pi_{n}(A)^{(\gamma(e))}\right] \mathbf{L}^{-(n+1) d}=\left[\pi_{n^{\prime}}(A)^{(\gamma(e))}\right] \mathbf{L}^{-\left(n^{\prime}+1\right) d} .
$$

We deduce that, for $n$ and $n^{\prime} \geq c \gamma(e),\left[\pi_{n}(A)\right] \mathbf{L}^{-(n+1) d}-\left[\pi_{n^{\prime}}(A)\right] \mathbf{L}^{-\left(n^{\prime}+1\right) d}$ belongs to $F^{e+1} \mathcal{M}_{\text {loc }}$. This implies that $\left[\pi_{n}(A)\right] \mathbf{L}^{-(n+1) d}$ is a Cauchy sequence, hence converges to a limit $\mathcal{A}$ in $\widehat{\mathcal{M}}$. Since, by $(3.2 .1), \mu\left(A^{(\gamma(e))}\right)=\left[\pi_{n}\left(A^{(\gamma(e))}\right)\right] \mathbf{L}^{-(n+1) d}$ when $n \geq$ $c \gamma(e)$, it follows that $\mu\left(A^{(\gamma(e))}\right)$ converges to $\mathcal{A}$ in $\widehat{\mathcal{M}}$, and we deduce that $\mathcal{A}=\mu(A)$, since, by (3.2.3), $\mu\left(A^{(\gamma(e))}\right)$ converges to $\mu(A)$. Because $\gamma(0)=0$ and $\pi_{0}\left(\mathbf{A}^{(0)}\right)=$ $\pi_{0}(A) \backslash S$, we obtain from (1) and (2) (with $e=0, n^{\prime}=0, n \rightarrow \infty$ ) that $\mu(A)-$ $\left[\pi_{0}(A) \backslash S\right] \mathbf{L}^{-d}$ belongs to $F^{1} \widehat{\mathcal{M}}$. When $A=\mathcal{L}(X)$, we obtain that $\mu(\mathcal{L}(X))$ is nonzero, since $[X \backslash S] \mathbf{L}^{-d} \notin F^{1} \widehat{\mathcal{M}}$ (this last fact may be checked by considering the degree of Hodge polynomials, cf. (6.1)).

Remark. - It follows from Theorem 7.1 and Lemma 4.3 that, for any algebraic variety $X$ over $k$ of dimension $d$, and any semi-algebraic subset $A$ of $\mathcal{L}(X)$, $\left[\pi_{n}(A)\right] \mathbf{L}^{-(n+1) d}$ converges in $\widehat{\mathcal{M}}$ to $\mu_{\mathcal{L}\left(X_{d}\right)}\left(A \cap \mathcal{L}\left(X_{d}\right)\right)$, where $X_{d}$ is the union of the $d$-dimensional components of $X$.

\section{Remarks on the Greenberg function}

Let $X$ be an algebraic variety over $k$. The Greenberg function $n \mapsto \gamma(n)$ for $X$ has been defined in 4.4. For $n \geq m$, we denote by $\pi_{n, m}$ the canonical morphism $\mathcal{L}_{n}(X) \rightarrow \mathcal{L}_{m}(X)$. We set $X_{n, j}:=\pi_{n+j, n} \mathcal{L}_{n+j}(X)$ and consider the Poincaré series

$$
P(T, U)=\sum_{n, j \in \mathbf{N}}\left[X_{n, j}\right] T^{n} U^{j}
$$

as an element of $\mathcal{M}_{\text {loc }}[[T, U]]$.

The following result is an analogue of [D1] Theorem 5.1 and Proposition 5.2. 
THEOREM 8.1. - (1) The series $P(T, U)$ is rational and can be written as

$$
P(T, U)=q(T, U)(1-U)^{-1} \prod_{i=1}^{e}\left(1-\mathbf{L}^{a_{i}} T^{b_{i}} U^{c_{i}}\right)^{-1}
$$

with $q(T, U)$ in $\mathcal{M}_{\mathrm{loc}}[T, U], a_{i}$ in $\mathbf{Z}, b_{i}, c_{i}$ in $\mathbf{N}$, and $b_{i} \geq 1$.

(2) Suppose none of the factors $1-\mathbf{L}^{a_{i}} T^{b_{i}} U^{c_{i}}$ divides $q(T, U)$ in $\mathcal{M}_{\mathrm{loc}}[T, U]$. Then $c:=1+\sup _{i} c_{i} / b_{i}$ is the smallest real number such that there exists $d$ in $\mathbf{R}$ with $\gamma(n) \leq c n+d$ for all $n$ in $\mathbf{N}$.

Proof. - The proof is very much the same as the one in [D1]. By a similar proof as the one of Theorem 1.1 one obtains that $P(T, U)$ has the form required in (1), except for the fact that $b_{i} \geq 1$, which is proved in the same way as in [D1]. The proof of (2) is also similar to the one of [D1]. However we have to use that the image in $\mathcal{M}_{\text {loc }}$ of a non-empty constructible subset of an algebraic variety is nonzero, a fact that may be checked by considering the degree of Hodge polynomials, cf. (6.1).

Remark. - It follows from the result of Pas (Theorem 2.1) that there exists a finite partition of $\mathbf{N}$ into congruences classes, such that the function $\gamma$ is linear on each class for $n$ large enough.

\section{References}

[A-K] J. Ax, S. Kochen, Diophantine problems over local fields III, Decidable fields, Ann. Math. 83 (1966), 437-456.

[B-D] V. V. BAtyrev, D. I. DAIs, Strong McKay correspondence, stringtheoretic Hodge numbers and mirror symmetry, Topology 35 (1996), 901-929.

[B-L-R] S. Bosch, W. Lütkebohmert, M. Raynaud, Néron Models, Ergeb. Math. Grenzgeb. (3) 21, Springer-Verlag, Berlin, 1990.

[C-K] C. C. Chang, H. J. Keisler, Model Theory, North-Holland, 1973.

[Da] V. I. DAnilov, The geometry of toric varieties, Russian Math. Surveys 33 (1978), 97-154.

[De] F. Delon, Quelques propriétés des corps valués, Thèse d'État, Université Paris VII (1981).

[D1] J. Denef, On the rationality of the Poincaré series associated to the p-adic points on a variety, Invent. math. 77 (1984), 1-23. 
[D2] J. DEnEF, On the evaluation of certain p-adic integrals, Séminaire de Théorie des Nombres, Paris 1983-84, Progress in Math. 59, Birkhäuser (1985), 25-47.

[D-L] J. Denef, F. Loeser, Motivic Igusa zeta functions, to appear in Journal of Algebraic Geometry.

[G-S] H. Gillet, C. Soulé, Descent, motives and K-theory, J. reine angew. Math. 478 (1996), 127-176.

[G] M. Greenberg, Rational points in henselian discrete valuation rings, Publ. Math. I.H.E.S. 31 (1966), 59-64.

[G-D] A. Grothendieck, J.A. Dieudonné, Eléments de Géométrie Algébrique I, Grundlehren Math. Wiss. 166, Springer-Verlag, Berlin, 1971.

[G-N] F. Guillén, V. NAVArro Aznar, Un critère d'extension d'un foncteur défini sur les schémas lisses, preprint (1995), revised (1996).

[H] M. Hickel, Fonction de Artin et germes de courbes tracées sur un germe d'espace analytique, Amer. J. Math. 115 (1993), 1299-1334.

[K] M. Kontsevich, Lecture at Orsay (December 7, 1995).

[L-J] M. LEJeune-JALABERT, Courbes tracées sur un germe d'hypersurface, Amer. J. Math. 112 (1990), 525-568.

[N] J.F. NASH JR., Arc structure of singularities, Duke Math. Journ. 81 (1995), 31-38.

[O] J. OESTERLÉ, Réduction modulo $p^{n}$ des sous-ensembles analytiques fermés de $\mathbf{Z}_{p}^{N}$, Invent. math. 66 (1982), 325-341.

[P] J. PAS, Uniform p-adic cell decomposition and local zeta functions, J. reine angew. Math. 399 (1989), 137-172.

[Pr] M. Presburger, Uber die Vollständigkeit eines gewissen Systems des Arithmetik ..., Comptes-rendus du I Congrès des Mathématiciens des Pays Slaves, Warsaw (1929), 92-101.

[V] W. VEYs, The topological zeta function associated to a function on a normal surface germ, preprint.

J.D. University of Leuven, Department of Mathematics, Celestijnenlaan 200B, 3001 Leuven, Belgium

Jan. Denef@wis . kuleuven . ac . be

F.L. Centre de Mathématiques, Ecole Polytechnique, F-91128 Palaiseau (URA 169 du CNRS)

et 
Institut de Mathématiques, Université P. et M. Curie, Case 82, 4 place Jussieu, F-75252 Paris Cedex 05 (UMR 9994 du CNRS)

loeser@math. polytechnique.fr 